\title{
MULTIPLE ORTHOGONAL GEODESIC CHORDS IN NONCONVEX RIEMANNIAN DISKS USING OBSTACLES
}

\author{
ROBERTO GIAMBÒ, FABIO GIANNONI, PAOLO PICCIONE
}

\begin{abstract}
We use nonsmooth critical point theory and the theory of geodesics with obstacle to show a multiplicity result about orthogonal geodesic chords in a Riemannian manifold (with boundary) which is homeomorphic to an $\mathrm{N}$-disk. This applies to brake orbits in a potential well of a natural Hamiltonian system, providing a further step towards the proof of a celebrated conjecture by Seifert [26].
\end{abstract}

\section{INTRODUCTION}

Let $(\bar{\Omega}, g)$ be a compact smooth $\left(\mathrm{C}^{3}\right)$ Riemannian manifold with smooth $\left(\mathrm{C}^{2}\right)$ boundary $\partial \Omega$; denote by $\Omega=\bar{\Omega} \backslash \partial \Omega$. A geodesic chord in $(\bar{\Omega}, \mathrm{g})$ is a geodesic $\gamma:[0,1] \rightarrow \bar{\Omega}$ with $\gamma(0), \gamma(1) \in \partial \Omega$ and $\gamma(s) \in \Omega$ for any $s \in] 0,1$. An orthogonal geodesic chord (OGC, for shortness) is a geodesic chord $\gamma$ in $(\bar{\Omega}, g)$ with $\dot{\gamma}(0)$ and $\dot{\gamma}(1)$ orthogonal to $\partial \Omega$ at $\gamma(0)$ and $\gamma(1)$ respectively.

In this paper, we will give a multiplicity result for OGC's in the case where $\bar{\Omega}$ is homeomorphic to an $\mathrm{N}$-dimensional disk $\mathbb{D}^{\mathrm{N}} \subset \mathbb{R}^{\mathrm{N}}$; manifolds of this type will be called Riemannian $\mathrm{N}$-disks. When $\bar{\Omega}$ is a convex body of $\mathbb{R}^{\mathrm{N}}$, then a classical result by Lusternik and Schnirelman [21] gives the existence of at least N segments contained in $\bar{\Omega}$ that meet $\partial \Omega$ orthogonally at both endpoints. This result has been generalized by Bos [1], who proved the existence of at least N orthogonal geodesic chords when $\bar{\Omega}$ is a convex domain of an arbitrary Riemannian $\mathrm{N}$-manifold. The convexity assumption is crucial for these results, as it allows to use shortening methods.

Currently, the real challenge is to go beyond the convex case, and consider situations where two arbitrary points in $\Omega$ are not necessarily joined by a minimal geodesic in $\bar{\Omega}$, or, similarly, when a geodesic with endpoints in $\Omega$ may be somewhere tangent to $\partial \Omega$. We will think of this situation as if $\partial \Omega$ were an obstacle to the geodesical connectedness of $\bar{\Omega}$, or to the existence of OGC's in $\bar{\Omega}$.

The interest in this situation arises, mainly, from a famous old conjecture due to Seifert [26] on the minimal number of periodic orbits (brake orbits) of a Hamiltonian system with fixed energy in a potential well. Using Maupertuis's principle, such periodic orbits can be described in terms of orthogonal geodesics chords of a Riemannian disk, whose boundary is strictly concave, cf. [10]. The proof of this conjecture has motivated an intense literature on the subject (cf. e.g. [23]). In spite of the fact that a complete proof of the conjecture has not been reached yet, important contributions were given by several researchers, including Long's school [17, 18, 19, 20, 28, 29, 30], among many others (cf. also [14]).

The present paper aims at giving a further step towards a positive answer to Seifert's conjecture, with the proof of the existence of multiple OGC's in Riemannian disks with

Date: January 22nd, 2018.

2000 Mathematics Subject Classification. 37C29, 37J45, 58E10. 
possibly non-convex boundary, but satisfying a mild global condition that can be described as follows. A geodesic segment $\gamma:[0, \mathrm{~b}] \rightarrow \bar{\Omega}$ is an $O-T$ chord (orthogonal-tangent) if $\gamma(0), \gamma(\mathrm{b}) \in \partial \Omega, \dot{\gamma}(0)$ is orthogonal to $\partial \Omega$ and $\dot{\gamma}(\mathrm{b})$ is tangent to $\partial \Omega$. The central assumption of our main theorem is that $(\bar{\Omega}, \mathrm{g})$ does not admit any O-T geodesic. Two OGC's $\gamma_{1}, \gamma_{2}:[0,1] \rightarrow \bar{\Omega}$ will be considered distinct when $\gamma_{1}([0,1]) \neq \gamma_{2}([0,1])$. Our main result has the following statement.

Theorem 1.1. Let $(\bar{\Omega}, \mathrm{g})$ be a Riemannian $\mathrm{N}$-disk without $O-T$ chords. Then, there are at least $\mathrm{N}$ distinct orthogonal geodesic chords in $\bar{\Omega}$.

The proof of the above results presented here employs two techniques:

- a non-smooth critical point theory;

- the theory of geodesics in manifolds with obstacles.

Note that the above result could be obtained using a geometrical approach based on a shortening method for geodesics on manifolds with boundary. The related uniqueness result of the minimizing geodesic connecting two close points was proved in [25]. Here, instead, we use a variational approach, considering the geodesic action functional in the set of $H^{1}$-curves in $\bar{\Omega}$ having free endpoints in $\partial \Omega$. The standard modeling over the Hilbert space $H^{1}\left([0,1], \mathbb{R}^{N}\right)$ does not provide a differentiable structure on such a set: paths that are somewhere tangent to $\partial \Omega$ are singular points of this set. The notion of criticality for this variational setup follows the theory of the weak slope (cf. [3, 4, 7]), which gives us the deformation lemmas (see Section 5).

The theory of geodesics with obstacle (namely curves having second derivative in $\mathrm{L}^{\infty}$ satisfying equation (3.11) below) developed in the pioneering work of Marino and Scolozzi [22] for geodesics having fixed endpoints, is used in a crucial way to show the $C^{1}$-regularity of the critical points, while the assumption of absence of $\mathrm{O}-\mathrm{T}$ chords is used to show that critical points at a positive critical level correspond indeed to OGC's in $\bar{\Omega}$ (Proposition 3.5).

Multiplicity of gedesics in a non-contractible Riemannian manifold $M$ with boundary, connecting submanifolds with boundary $M_{0}$ and $M_{1}$, was studied in [24]. Assuming $M_{0}$ and $M_{1}$ contractible in $M$, the authors obtain the existence of infinitely many geodesics. Such a result has been improved in [2] with weaker regularity assumptions on $M$, which is assumed only to be a p-convex set. In both the above papers, the authors employ minimax methods, using the theory of curves of maximal slope, (cf. e.g. [5, 6]).

A multiplicity result in an annulus (at least two orthogonal geodesic chords) has been proved in [12], together with appplications to multiplicity results for brake orbits and homoclinics. Then in [13] examples of potentials with exactly two brake orbits and two homoclinics are given.

A few remarks are in order. First, let us observe that the absence of O-T chords in $\bar{\Omega}$ is a condition which is open w.r.t. the $C^{1}$-topology in the set of smooth Riemannian metrics in $\bar{\Omega}$. It is in particular satisfied when $\mathrm{g}$ is a metric with rotational symmetry around some point $\mathrm{q} \in \Omega$, in which case all chords that start orthogonally from $\partial \Omega$ also arrive orthogonally onto $\partial \Omega$. Thus, our theorem guarantees the existence of at least $\mathrm{N}$ distinct OGC's when $\mathrm{g}$ is obtained from a small $\mathrm{C}^{1}$-perturbation of a rotationally symmetric metric in $\Omega$.

It should also be observed that the absence of $\mathrm{O}-\mathrm{T}$ chords is not a generic condition. It is rather intuitive that one can construct examples of metrics on the disk admitting O-T chords, and such that the existence of O-T chords persists after sufficiently small perturbations of the metric. We will give a formal proof of this fact in Appendix A using a transversality argument. Nevertheless, also the existence of multiple OGC's is not a generic 
property for metrics on the disk, by the same type of arguments, so that the assumption in our Theorem is perfectly compatible with the problem.

Let us also remark that our result allows to improve the multiplicity result obtained in [15] concerning small perturbations of the radial case.

Using the relation between orthogonal geodesic chords and brake orbits of a natural Lagrangian (or Hamiltonian) system (cf. [10]), Theorem 1.1 gives a partial answer to the above mentioned conjecture of Seifert. Let us use the following terminology: given a Riemannian manifold $(M, g)$ and a smooth function $V: M \rightarrow \mathbb{R}$, we say that the triple $(\mathrm{M}, \mathrm{g}, \mathrm{V})$ is a data for the Lagrangian system:

$$
\frac{\mathrm{D}}{\mathrm{dt}} \dot{\mathrm{x}}=-\nabla \mathrm{V}(\mathrm{x}),
$$

where $\frac{D}{d t}$ denotes the covariant derivative operator along the curve $x:[0, T] \rightarrow M$ relative to the Levi-Civita connection of $\mathrm{g}$. Let us recall that brake orbits for the Lagrangian system form a special class of periodic solutions of (1.1).

Theorem 1.2. Let $\left(M, g_{0}\right)$ be a Riemannian $N$-manifold whose metric is rotationally symmetric around some point $\mathrm{q}_{0} \in M$. Let $\mathrm{V}_{0}: M \rightarrow \mathbb{R}$ be a smooth function, which is also rotationally invariant around $\mathrm{q}_{0}$, and let $\mathrm{E}_{0} \in \mathbb{R}$ be a regular value of $\mathrm{V}_{0}$; assume that the sublevel $\left.\left.\mathrm{V}_{0}^{-1}(]-\infty, \mathrm{E}_{0}\right]\right)$ is homeomorphic to the $\mathrm{N}$-disk. Then, for any $\mathrm{C}^{2}$-metric $\mathrm{g}$ and $\mathrm{C}^{2}$-function $\mathrm{V}$ sufficiently $\mathrm{C}^{1}$-close to $\mathrm{g}_{0}$ and $\mathrm{V}_{0}$ respectively, and for all $\mathrm{E} \in \mathbb{R}$ sufficiently close to $\mathrm{E}_{0}$, there are at least $\mathrm{N}$ geometrically distinct brake orbits of energy $\mathrm{E}$ for the Lagrangian system with data $(\mathrm{M}, \mathrm{g}, \mathrm{V})$.

Note that for the above result we do not need nondegeneracy assumptions. Observe also that the famous result of Bos ([1]) about multiplicity of OGC's in the convex case becomes a particular case of our Theorem 1.1.

Acknowledgment. The authors wish to thank Marco Degiovanni for useful discussions and suggestions concerning the weak slope theory and the study of Palais-Smale sequences.

\section{A NONSMOOTH FUNCTIONAL FRAMEWORK}

It will be convenient to assume that $\bar{\Omega}$ is isometrically embedded into a larger open Riemannian $N$-manifold $(M, g)$. In this situation, $\Omega$ is open in $M$, its closure in $M$ coincides with $\bar{\Omega}$ and its boundary in $M$ is $\partial \Omega$. Using the Whitney Embebbing Theorem (cf [27]), we can assume that $M$ is an embedded submanifold of $\mathbb{R}^{2 N}$. This will provide a useful linear structure to our framework. Thus, one has inclusions:

$$
\bar{\Omega} \subset M \subset \mathbb{R}^{2 N} \text {. }
$$

2.1. Geometry of $\overline{\mathbf{\Omega}}$. Let us fix a $C^{2}$-function $\phi: M \rightarrow \mathbb{R}$ with the property that $\Omega=$ $\phi^{-1}(]-\infty, 0[)$ and $\partial \Omega=\phi^{-1}(0)$, with $\nabla \phi \neq 0$ on $\partial \Omega$. One can choose $\phi$ such that, in a neighborhood of $\partial \Omega$

$$
\phi(x)= \begin{cases}-\operatorname{dist}(q, \partial \Omega), & \text { if } x \in \bar{\Omega} \\ \operatorname{dist}(q, \partial \Omega), & \text { if } x \notin \bar{\Omega} .\end{cases}
$$

Let us also fix $\delta_{0}>0$ small enough so that:

$$
\nabla \phi(p) \neq 0 \text { for any } p \in \phi^{-1}\left(\left[-\delta_{0}, \delta_{0}\right]\right) ;
$$

thus, the set $\phi^{-1}\left(\left[-\delta_{0}, \delta_{0}\right]\right)$ is a tubular neighborhood of $\partial \Omega$, and there exists a continuous retraction:

$$
\mathbf{r}: \phi^{-1}\left(\left[-\delta_{0}, \delta_{0}\right]\right) \longrightarrow \partial \Omega
$$


defined in terms of the flow of $\nabla \phi$. The normalized gradient of $\phi$ :

$$
v(p)=\frac{\nabla \phi(p)}{\|\nabla \phi(p)\|}, \quad p \in \partial \Omega,
$$

gives a unit normal vector field along $\partial \Omega$ which points outwards. It will be useful to consider a fixed $C^{1}$-extension of $v$ to $M$. The notions of convexity, or concavity, for $\bar{\Omega}$ can be given in terms of the second fundamental form of $\partial \Omega$ or, equivalently, in terms of the Hessian $\mathrm{H}^{\phi}$ of the function $\phi: \partial \Omega$ is convex (resp., concave) when for all $p \in \partial \Omega, \mathrm{H}^{\phi}$ is positive semidefinite (resp., negative semidefinite) on $T_{p} \partial \Omega$.

Let us also define the positive constant $\mathrm{K}_{0}$ as follows:

$$
\mathrm{K}_{0}=\max _{\mathrm{x} \in \bar{\Omega}}\|\nabla \phi(\mathrm{x})\| \text {. }
$$

2.2. Hilbert spaces and manifolds. For $[a, b] \subset \mathbb{R}$, let us consider the Sobolev spaces $\mathrm{H}^{1}\left([\mathrm{a}, \mathrm{b}], \mathbb{R}^{2 \mathrm{~N}}\right)$ and $\mathrm{H}_{0}^{1}\left([\mathrm{a}, \mathrm{b}], \mathbb{R}^{2 \mathrm{~N}}\right)=\left\{\mathrm{V} \in \mathrm{H}^{1}\left([\mathrm{a}, \mathrm{b}], \mathbb{R}^{2 \mathrm{~N}}\right): \mathrm{V}(\mathrm{a})=\mathrm{V}(\mathrm{b})=0\right\}$. For $S \subseteq M$, set:

$$
H^{1}([a, b], S)=\left\{x \in H^{1}\left([a, b], \mathbb{R}^{2 N}\right): x(s) \in S \text { for all } s \in[a, b]\right\} .
$$

In particular, $H^{1}([a, b], M)$ is a smooth Hilbert submanifold of $H^{1}\left([a, b], \mathbb{R}^{2 N}\right)$ modeled on the Hilbert space $H^{1}\left([a, b], \mathbb{R}^{N}\right)$. For $x \in H^{1}([a, b], M)$, the tangent space $T_{x} H^{1}([a, b], M)$ is identified with the Hilbert subspace of $H^{1}\left([a, b], \mathbb{R}^{2 N}\right)$ given by:

$$
\mathrm{T}_{x} H^{1}([a, b], M)=\left\{\xi \in H^{1}\left([a, b], \mathbb{R}^{2 N}\right): \xi(s) \in T_{x(s)} M \quad \text { for all } s \in[a, b]\right\} .
$$

We will define some distance functions on $H^{1}([0,1], M)$ by setting:

$$
\begin{aligned}
\operatorname{dist}_{*}\left(x_{1}, x_{2}\right)=\max \left\{\left\|x_{2}(0)-x_{1}(0)\right\|_{2 N}, \|\right. & \left.x_{2}(1)-x_{1}(1) \|_{2 N}\right\}+ \\
& \left(\int_{0}^{1}\left\|\dot{x}_{2}(s)-\dot{x}_{1}(s)\right\|_{2 N}^{2} \mathrm{ds}\right)^{\frac{1}{2}},
\end{aligned}
$$

and

$$
\operatorname{dist}_{\infty}\left(x_{1}, x_{2}\right)=\sup _{s \in[0,1]}\left\|x_{2}(s)-x_{1}(s)\right\|_{2 N} .
$$

Here, $\|\cdot\|_{2 \mathrm{~N}}$ is the Euclidean norm in $\mathbb{R}^{2 \mathrm{~N}}$. Note that,

$$
\operatorname{dist}_{\infty}\left(x_{1}, x_{2}\right) \leqslant \operatorname{dist}_{*}\left(x_{1}, x_{2}\right), \quad \forall x_{1}, x_{2} \in H^{1}([0,1], M) .
$$

Finally, we consider the norm $\|\cdot\|_{*}$ in $H^{1}\left([0,1], \mathbb{R}^{2 N}\right)$ defined by:

$$
\|\mathrm{V}\|_{*}=\max \{\|\mathrm{V}(0)\|,\|\mathrm{V}(1)\|\}+\left(\int_{\mathrm{a}}^{\mathrm{b}}\left\|\mathrm{V}^{\prime}\right\|^{2} \mathrm{ds}\right)^{\frac{1}{2}} \text {. }
$$

Observe that all the norms and distances defined here are invariant by backward reparameterizations of curves, see Section 2.4.

2.3. Functional space and the energy functional. The main functional space considered for our variational problem is:

$$
\mathfrak{M}=\left\{x \in H^{1}([0,1], \bar{\Omega}): x(0), x(1) \in \partial \Omega\right\},
$$

endowed with the usual energy functional

$$
\mathcal{F}(x)=\int_{0}^{1} g(\dot{x}, \dot{x}) d s .
$$


This is a smooth functional on the Hilbert manifold $H^{1}([0,1], M)$, and its restriction to $\mathfrak{M}$ is clearly continuous. For $x \in H^{1}([0,1], M)$ and $V \in T_{x} H^{1}([0,1], M)$, the derivative $\mathrm{d} \mathcal{F}(\mathrm{x}) \mathrm{V}$ is given by the integral formula:

$$
\mathrm{d} \mathcal{F}(\mathrm{x}) \mathrm{V}=2 \int_{0}^{1} \mathrm{~g}\left(\dot{\mathrm{x}}, \frac{\mathrm{D}}{\mathrm{d} s} \mathrm{~V}\right) \mathrm{ds},
$$

where $\frac{\mathrm{D}}{\mathrm{ds}}$ denotes the covariant derivative along $x$ relatively to the Levi-Civita connection of $g$.

Lemma 2.1. Let $x \in \mathfrak{M}$ and $[a, b] \subset[0,1]$ be such that $x(a) \in \partial \Omega$. Then

$$
\max _{s \in[a, b]} \mid \phi\left(x(s) \mid \leqslant K_{0}(b-a)^{\frac{1}{2}}\left[\int_{a}^{b} g(\dot{x}, \dot{x}) d \sigma\right]^{\frac{1}{2}} .\right.
$$

Proof. Since $\phi(x(a))=0$ and $\|\nabla \phi(x)\| \leqslant K_{0}$ for any $x \in \bar{\Omega}$, for all $s \in[a, b]$ we have

$$
\begin{aligned}
|\phi(x(s))|=|\phi(x(s))-\phi(x(a))| & \leqslant \int_{a}^{s}|g(\nabla \phi(x(\sigma)), \dot{x}(\sigma))| d \sigma \\
& \leqslant K_{0} \int_{a}^{s} g(\dot{x}, \dot{x})^{\frac{1}{2}} d \sigma \leqslant K_{0} \sqrt{s-a}\left(\int_{a}^{b} g(\dot{x}, \dot{x}) d \sigma\right)^{\frac{1}{2}},
\end{aligned}
$$

from which (2.14) follows.

Corollary 2.2. Let $\chi \in \mathfrak{M}$ and $[\mathrm{a}, \mathrm{b}] \subset[0,1]$ be such that $\chi(\mathrm{a}), \chi(\mathrm{b}) \in \partial \Omega$ and

$$
\int_{a}^{b} g(\dot{x}, \dot{x}) d \sigma \leqslant \frac{\delta_{0}^{2}}{K_{0}^{2}}
$$

Then, $\phi(x(s)) \geqslant-\delta_{0}$ for all $s \in[a, b]$.

2.4. Backward reparameterization. Consider now the map $\mathcal{R}: \mathfrak{M} \rightarrow \mathfrak{M}$ :

$$
\mathcal{R} x(t)=x(1-t) .
$$

We say that $\mathcal{N} \subset \mathfrak{M}$ is $\mathcal{R}$-invariant if $\mathcal{R}(\mathcal{N})=\mathcal{N}$; note that $\mathfrak{M}$ is $\mathcal{R}$-invariant. If $\mathcal{N}$ is $\mathcal{R}$-invariant, a homotopy $\mathrm{h}:[0,1] \times \mathcal{N} \rightarrow \mathfrak{M}$ is called $\mathcal{R}$-equivariant if

$$
h(\tau, \mathcal{R} x)=\mathcal{R h}(\tau, x), \quad \forall x \in \mathcal{N}, \forall \tau \in[0,1] .
$$

Remark 2.3. Note that if $\gamma:[0,1] \rightarrow \bar{\Omega}$ is an OGC, then $\gamma$ and $\mathcal{R} \gamma$ are not distinct OGC's according to our definition, however, $\mathcal{R} \gamma \neq \gamma$ as elements of $\mathfrak{M}$. Indeed if by contradiction $\mathcal{R} \gamma=\gamma$, i.e., $\gamma(1-t)=\gamma(\mathrm{t})$ for all $\mathrm{t}$, and from this it would follow $\dot{\gamma}\left(\frac{1}{2}\right)=0$. Since $\mathrm{g}(\dot{\gamma}, \dot{\gamma})$ is constant, we would then have that $\gamma$ is constant.

2.5. The set of chords of $\bar{\Omega}$. The following Lemma allows to describe an $\mathcal{R}$-invariant subset $\mathfrak{C}$ of $\mathfrak{M}$ which is homeomorphic to the product $\mathbb{S}^{N-1} \times \mathbb{S}^{N-1}$. This is interpreted as the set of chords in $\bar{\Omega}$.

Lemma 2.4. There exists a continuous map $\gamma: \partial \Omega \times \partial \Omega \rightarrow H^{1}([0,1], \bar{\Omega})$ such that

(1) $\gamma(A, B)(0)=A, \gamma(A, B)(1)=B$.

(2) $\mathrm{A} \neq \mathrm{B} \Rightarrow \gamma(\mathrm{A}, \mathrm{B})(\mathrm{s}) \in \Omega \forall s \in] 0,1[$.

(3) $\gamma(A, A)(s)=A \forall s \in[0,1]$.

(4) $\mathcal{R} \gamma(A, B)=\gamma(B, A)$, namely $\gamma(A, B)(1-s)=\gamma(B, A)(s)$ for all s, and for all $A, B$. 
Proof. Let $\Psi: \bar{\Omega} \rightarrow \mathbb{D}^{N}$ be a homeomorphism. Define

$$
\hat{\gamma}(A, B)(s)=\Psi^{-1}((1-s) \Psi(A)+s \Psi(B)), A, B \in \bar{\Omega} .
$$

In general, if $\bar{\Omega}$ is only homeomorphic to the disk $\mathbb{D}^{N}$, the above definition produces curves that in principle are only continuous. In order to produce curves with an $\mathrm{H}^{1}$-regularity, it suffices to use a broken geodesic approximation argument.

After choosing a function $\gamma: \partial \Omega \times \partial \Omega \rightarrow H^{1}([0,1], \bar{\Omega})$ as in Lemma 2.4, we set:

$$
\begin{aligned}
& \mathfrak{C}=\{\gamma(A, B): A, B \in \partial \Omega\}, \\
& \mathfrak{C}_{0}=\{\gamma(A, A): A \in \partial \Omega\} .
\end{aligned}
$$

Remark 2.5. Note that the map $\partial \Omega \times \partial \Omega \ni(A, B) \mapsto \gamma(A, B) \in \mathfrak{C}$ is a homeomorphism.

Using the continuity of $\mathcal{F}$ and the compactness of $\mathfrak{C}$, let us define $M_{0} \in \mathbb{R}^{+}$by:

$$
M_{0}^{2}=\sup _{x \in \mathfrak{C}} \int_{0}^{1} g(\dot{x}, \dot{x}) d t .
$$

Remark 2.6. It is interesting to observe that the constants $\delta_{0}$ in (2.2), $\mathrm{K}_{0}$ in (2.5) and $M_{0}$ in (2.18) are related by the following inequality:

$$
\mathrm{M}_{0}>\frac{\delta_{0}}{\mathrm{~K}_{0}} .
$$

Indeed assume by contradiction $M_{0} \leqslant \frac{\delta_{0}}{K_{0}}$. By Corollary 2.2, all the curves of $\mathfrak{C}$ must then lie in the tubular neighborhood $\phi^{-1}\left(\left[-\delta_{0}, \delta_{0}\right]\right)$ of $\partial \Omega$. Using the retraction $\mathbf{r}$ defined in (2.3), one could then obtain a retraction $\mathbf{r}_{0}$ of $\partial \Omega \times \partial \Omega$ onto its diagonal, by defining $\mathbf{r}_{0}(A, B)$ as the midpoint of the path $\mathbf{r} \circ \gamma(A, B)$. Of course, such a retraction cannot exist, which leads to a contradiction and proves (2.19).

\section{ON THE $\mathcal{V}^{-}$-CRITICAL CURVES OF THE ENERGY FUNCTIONAL $\mathcal{F}$ ON $\mathfrak{M}$}

Given $x \in \mathfrak{M}$, let us define the notion of admissible infinitesimal variation of $\chi$ in $\mathfrak{M}$ as a vector field $V \in T_{x} H^{1}([0,1], M)$ satisfying:

and

$$
g(\nabla \phi(x(0)), \mathrm{V}(0))=g(\nabla \phi(x(1)), \mathrm{V}(1))=0,
$$

Since $\nabla \phi(p)$ points outside of $\Omega$ for $p \in \partial \Omega$, then condition (3.2) says that $V(s)$ must not point outside $\Omega$ when $x(s) \in \partial \Omega$. The set of admissible infinitesimal variations of $x$ in $\mathfrak{M}$ will be denoted by $\mathcal{V}^{-}(x)$.

In view of the fact that $\mathfrak{M}$ is not smooth, we need a suitable definition of critical points for the functional $\mathcal{F}: \mathfrak{M} \rightarrow \mathbb{R}$. Following the weak slope theory developed in [3, 7], and recalling (2.13), we give the following.

Definition 3.1. We say that $x$ is a $\mathcal{V}^{-}$-critical curve for $\mathcal{F}$ on $\mathfrak{M}$ if

$$
\int_{0}^{1} g\left(\dot{x}, \frac{D}{d s} V\right) d s \geqslant 0, \quad \text { for all } V \in \mathcal{V}^{-}(x) .
$$

Note that the set $\mathcal{F}^{-1}(0)$ consists entirely of minimum points (the constant curves in $\partial \Omega$ ) which are obviously $\mathcal{V}^{-}$-critical curves. In order to describe the $\mathcal{V}^{-}$-critical curves of $\mathcal{F}$ corresponding to positive critical levels, we start with the following regularity result proved in [22] in an $\mathrm{L}^{2}$ setting. Here we give a simpler proof in an $\mathrm{H}^{1}$ setting. 
Proposition 3.2. Let $z \in \mathfrak{M}$ be a $\mathcal{V}^{-}$-critical curve for $\mathcal{F}$. Then $\frac{\mathrm{D}}{\mathrm{ds}} \dot{z} \in \mathrm{L}^{\infty}\left([0,1], \mathbb{R}^{2 \mathrm{~N}}\right)$ and, in particular, $z$ is of class $\mathrm{C}^{1}$.

Proof. Let $v$ be a $C^{1}$-vector field in $M$ as in (2.4). Given $\tau \in \mathbb{R}$, we will write $\tau^{+}$for $\max \{0, \tau\}$. For an arbitrary $\xi \in H_{0}^{1}\left([0,1], \mathbb{R}^{2 N}\right)$ vector field along $z$, set $\zeta=\xi-\eta$, where

$$
\eta(s)=g(\xi(s), v(z(s)))^{+} \cdot v(z(s)) .
$$

Observe that $\zeta \in \mathrm{H}_{0}^{1}\left([0,1], \mathbb{R}^{2 \mathrm{~N}}\right)$ and it satisfies (3.2), and therefore belongs to the set $\mathcal{V}^{-}(x)$ of admissible infinitesimal variations of $x$.

Let us set

$$
C_{z}=\{s \in[0,1]: \phi(z(s))=0\},
$$

and $\mathrm{I}_{z}=[0,1] \backslash \mathrm{C}_{z}$. Since $\mathrm{I}_{z}$ is open, it is a countable union of pairwise disjoint open intervals:

$$
\left.I_{z}=\bigcup_{i \in J}\right] a_{i}, b_{i}[
$$

where $\mathrm{J} \subset \mathbb{N}$. Then

$$
\int_{0}^{1} g\left(\dot{z}, \frac{\mathrm{D}}{\mathrm{dt}} \eta\right) \mathrm{d} s=\int_{\mathrm{C}_{z}} g\left(\dot{z}, \frac{\mathrm{D}}{\mathrm{dt}} \eta\right) \mathrm{d} s+\sum_{i \in J} \int_{\mathrm{a}_{i}}^{\mathrm{b}_{i}} \mathrm{~g}\left(\dot{z}, \frac{\mathrm{D}}{\mathrm{dt}} \eta\right) \mathrm{d} s .
$$

Let us consider an arbitrary vector field $\mathrm{V}$ of class $\mathrm{H}_{0}^{1}$ along $z$ such that $\left.\mathrm{V}\right|_{\mathrm{C}_{z}} \equiv 0$. Since both $\mathrm{V}$ and $-\mathrm{V}$ satisfy (3.1) and, trivially, also (3.2), we get

$$
\int_{a_{i}}^{b_{i}} g\left(\dot{z}, \frac{D}{d t} \eta\right) d s=0, \quad \forall i \in J
$$

Since $V$ is arbitrary outside $C_{z}, z_{\mid\left[a_{i}, b_{i}\right]}$ is a geodesic for all $i$. Partial integration gives

$$
\sum_{i \in J} \int_{a_{i}}^{b_{i}} g\left(\dot{z}, \frac{D}{d t} \eta\right) d s=\sum_{i \in J} g\left(\dot{z}\left(b_{i}\right), \eta\left(b_{i}\right)\right)-g\left(\dot{z}\left(a_{i}\right), \eta\left(a_{i}\right)\right) .
$$

But (3.4) ensures that, for all $s \in C_{z}, \eta(s)=\lambda(s) \nabla \phi(z(s))$ with $\lambda(s) \geqslant 0$, while

$$
g\left(\dot { z } ( b _ { i } ) , \nabla \phi ( z ( b _ { i } ) ) \geqslant 0 \text { , and } g \left(\dot{z}\left(a_{i}\right), \nabla \phi\left(z\left(a_{i}\right)\right) \leqslant 0 \quad \forall i \in J,\right.\right.
$$

because

Then, by (3.7),

$$
\phi(z(s)) \leqslant 0 \text { for all } s \in[a, b] .
$$

$$
\sum_{i \in J} \int_{a_{i}}^{b_{i}} g\left(\dot{z}, \frac{\mathrm{D}}{\mathrm{dt}} \eta\right) \mathrm{d} s \geqslant 0
$$

Since $\zeta=\xi-\eta$ satisfies (3.1) and (3.2) we also have

$$
\begin{aligned}
0 \leqslant \int_{0}^{1} g\left(\dot{z}, \frac{\mathrm{D}}{\mathrm{dt}} \xi\right) \mathrm{d} s-\int_{\mathrm{C}_{z}} \mathrm{~g}\left(\dot{z}, \frac{\mathrm{D}}{\mathrm{dt}} \eta\right) \mathrm{d} s & -\sum_{i \in J} \int_{\mathrm{a}_{i}}^{\mathrm{b}_{i}} \mathrm{~g}\left(\dot{z}, \frac{\mathrm{D}}{\mathrm{dt}} \eta\right) \mathrm{d} s \\
& \leqslant \int_{0}^{1} \mathrm{~g}\left(\dot{z}, \frac{\mathrm{D}}{\mathrm{dt}} \xi\right) \mathrm{d} s-\int_{\mathrm{C}_{z}} \mathrm{~g}\left(\dot{z}, \frac{\mathrm{D}}{\mathrm{dt}} \eta\right) \mathrm{d} s .
\end{aligned}
$$

Since $\phi \circ z$ is of class $\mathrm{H}^{1}$, and it is constant on $\mathrm{C}_{z}$, we have $\mathrm{g}(\dot{z}, \nabla \phi(z))=0$ a.e. on $\mathrm{C}_{z}$ (cf. [16]). Then

$$
\int_{C_{z}} g\left(\dot{z}, \frac{\mathrm{D}}{\mathrm{dt}} \eta\right) \mathrm{d} s=\int_{C_{z}} \mathrm{~g}\left(\dot{z}, \frac{\mathrm{D}}{\mathrm{ds}} v\right) g(\xi, v(z(s)))^{+} \mathrm{ds} .
$$


Then by (3.9) we get

$$
\int_{0}^{1} \mathrm{~g}\left(\dot{z}, \frac{\mathrm{D}}{\mathrm{dt}} \xi\right) \mathrm{d} s \geqslant \int_{\mathrm{C}_{z}} \mathrm{~g}\left(\dot{z}, \frac{\mathrm{D}}{\mathrm{d} s} v \dot{z}\right) \mathrm{g}(\xi, v)^{+} \mathrm{ds} .
$$

But $g(\dot{z}, \dot{z})$ is in $\mathrm{L}^{1}$, and then applying (3.10) to $\xi$ and $-\xi$, we obtain the existence of a constant $\mathrm{L}=\mathrm{L}(z)$ such that

$$
\left|\int_{0}^{1} \mathrm{~g}\left(\dot{z}, \frac{\mathrm{D}}{\mathrm{dt}} \xi\right) \mathrm{ds}\right| \leqslant \mathrm{L}(z)\|\xi\|_{\mathrm{L}^{\infty}}
$$

for any vector field $\xi$ of class $\mathrm{H}_{0}^{1}$, from which we deduce that $\dot{z}$ is in $\mathrm{L}^{\infty}$.

Then, using again (3.10) we deduce that there exists a constant $M=M(z)$ such that

$$
\left|\int_{0}^{1} g\left(\dot{z}, \frac{D}{d t} \xi\right) d s\right| \leqslant M(z)\|\xi\|_{L^{1}} .
$$

for any vector field $\xi$ along $z$ of class $\mathrm{H}_{0}^{1}$, from which the conclusion of the proof follows.

Lemma 3.3. Let $z \in \mathrm{H}^{1}([0,1], \bar{\Omega})$ be a $\mathcal{V}^{-}$-critical curve for $\mathcal{F}$ in $\mathfrak{M}$. Then $\mathrm{g}(\dot{z}, \dot{z})$ is constant and

$$
-\frac{\mathrm{D}}{\mathrm{ds}} \dot{z}=\lambda(\mathrm{s}) v(z(\mathrm{~s})) \text { a.e. },
$$

where $\lambda \in \mathrm{L}^{\infty}([0,1], \mathbb{R})$, and $\lambda(\mathrm{s})=0$ if $\phi(z(s))<0$. Moreover

$$
\lambda=\frac{g\left(\mathrm{H}^{\Phi}(z)[\dot{z}], \dot{z}\right)}{\sqrt{g(\nabla \phi(z), \nabla \phi(z))}} \text { a.e. in } \mathrm{C}_{z} \quad \text { (see (3.5)) }
$$

and

$$
\lambda(s) \leqslant 0 \text { a.e. }
$$

Proof. By Proposition 3.2, $\frac{\mathrm{D}}{\mathrm{ds}} \dot{z} \in \mathrm{L}^{\infty}$, so we can use partial integration in (3.3), obtaining

$$
\int_{0}^{1} \mathrm{~g}\left(-\frac{\mathrm{D}}{\mathrm{ds}} \dot{z}, \mathrm{~V}\right) \mathrm{d} s \geqslant 0, \quad \text { for all } \mathrm{V} \in \mathcal{V}^{-}(\mathrm{x}) \cap \mathrm{H}_{0}^{1}\left([0,1], \mathbb{R}^{\mathrm{N}}\right) .
$$

Then, $z$ is a free geodesic on any interval $\mathrm{I} \subset[0,1]$ such that $z(\mathrm{I}) \cap \partial \Omega=\emptyset$. Moreover, using vector fields $\mathrm{V}$ along $z$ such that $\mathrm{g}(\mathrm{V}(\mathrm{s}), \nabla \phi(z(\mathrm{~s})))=0$ for all $\mathrm{s}$ such that $z(\mathrm{~s}) \in$ $\partial \Omega$, we obtain

$$
-\frac{\mathrm{D}}{\mathrm{ds}} \dot{z}=\lambda(\mathrm{s}) \vee(z(\mathrm{~s})) \text { a.e., } \quad \text { for some } \lambda:[0,1] \rightarrow \mathbb{R} .
$$

Since $\frac{\mathrm{D}}{\mathrm{ds}} \dot{z}$ is in $\mathrm{L}^{\infty}$, then $\lambda \in \mathrm{L}^{\infty}([0,1], \mathbb{R})$, while $\lambda=0$ a.e. on $z^{-1}(\Omega)$.

Now, $z$ is of class $C^{1}$ and $\phi(z(s)) \leqslant 0$ for all $s \in[0,1]$. Therefore

$$
\mathrm{g}(\nabla \phi(z(\mathrm{~s})), \dot{z}(\mathrm{~s}))=0, \quad \text { for every } \mathrm{s} \in] 0,1[\text { such that } \phi(z(s))=0,
$$

and, contracting both terms of (3.15) with $\dot{z}$, we obtain that $\mathrm{g}(\dot{z}, \dot{z})$ is constant.

Now $\phi(z(s))=0$ for all $s \in C_{z}$. Therefore, differentiating twice and using once again [16], we obtain

$$
\mathrm{g}\left(\mathrm{H}^{\phi}(z(\mathrm{~s}))[\dot{z}(\mathrm{~s})], \dot{z}(\mathrm{~s})\right)+\mathrm{g}\left(\nabla \phi(z(\mathrm{~s})), \frac{\mathrm{D}}{\mathrm{ds}} \dot{z}(\mathrm{~s})\right)=0 \text { a.e. on } \mathrm{C}_{z} \text {. }
$$

Then contracting both terms of (3.11) with $\nabla \phi(z(s)$ we obtain (3.12).

It remains to prove (3.13). Towards this goal, apply formula (3.14) on any interval $[\alpha, \beta] \subset[0,1]$ with

$$
V_{n}(s)=-\chi_{n}(s) \nabla \phi(z(s)),
$$


where $\chi_{\mathrm{n}}$ is a sequence of positive bounded function, vanishing at $\alpha$ and $\beta$, and such that $\lim _{n \rightarrow \infty} \chi_{n}(s)=1$ for all $\left.s \in\right] \alpha, \beta\left[\right.$. Since $g\left(V_{n}, \nabla \phi(z)\right) \leqslant 0$ on $\partial \Omega$ for all $n$, we obtain

$$
\int_{\alpha}^{\beta} \lambda(s) g(\nabla \phi, v) d s \leqslant 0
$$

for all $[\alpha, \beta] \subset[0,1]$. From this, we deduce (3.13).

As to the endpoints of $\mathcal{V}^{-}$-critical curves, we have the following.

Lemma 3.4. If $z$ is a non-constant $\mathcal{V}^{-}$-critical curve of $\mathcal{F}$ in $\mathfrak{M}$, then $\dot{z}(0)$ and $\dot{z}(1)$ are orthogonal to $\partial \Omega$.

Proof. Take any vector field $\mathrm{V}$ along $z$ such that

$$
g(\nabla \phi(z(s)), V(s))=0 \text {, for any s such that } \phi(z(s))=0 .
$$

Using again the fact that, in this case, also $-\mathrm{V}$ is an admissible infinitesimal variation of $z$ in $\mathfrak{M}$, we obtain

$$
\int_{0}^{1} \mathrm{~g}\left(\dot{z}, \frac{\mathrm{D}}{\mathrm{d} s} \mathrm{~V}\right) \mathrm{ds}=0 .
$$

Then, partial integration and (3.11) give

$$
\mathrm{g}(\dot{z}(1), \mathrm{V}(1))-\mathrm{g}(\dot{z}(0), \mathrm{V}(0))=0 .
$$

Since $V(1)$ and $V(0)$ are arbitrary tangent vectors to $\partial \Omega$, we obtain desired conclusion.

We conclude this section with a complete description of the $\mathcal{V}^{-}$-critical curves of $\mathcal{F}$ in $\mathfrak{M}$.

Proposition 3.5. Assume that $(\bar{\Omega}, \mathrm{g})$ has no $O-T$ chords. If $\times$ is a $\mathcal{V}^{-}$-critical curve of $\mathcal{F}$ in $\mathfrak{M}$, with $\mathcal{F}(x)>0$, then $x$ is an $O G C$.

Proof. If $\mathcal{F}(x)>0$, then $x$ is a non-constant $\mathcal{V}^{-}$-critical curve with $\dot{x} \neq 0$ everywhere. By Lemma 3.4, $x$ starts orthogonally from $\partial \Omega$ at the instant $s=0$, moving inside $\Omega$. We need to show that $x(s) \in \Omega$ for all $s \in] 0,1[$. Assume by contradiction that this does not hold, and let $\left.s_{0} \in\right] 0,1\left[\right.$ be the first instant at which $x\left(s_{0}\right) \in \partial \Omega$. Since $x$ is of class $C^{1}$ (Proposition 3.2), then $\dot{x}\left(s_{0}\right)$ must be tangent to $\partial \Omega$. But then $x_{\left[0, s_{0}\right]}$ would be an O-T chord, which contradicts our assumption and proves the desired result.

The above results entitles us to give the following:

Definition 3.6. We say that $c>0$ is a $\mathcal{V}^{-}$-critical value for $\mathcal{F}: \mathfrak{M} \rightarrow \mathbb{R}$ if there exists a $\mathcal{V}^{-}$-critical curve $x$ such that $\mathcal{F}(x)=c$.

\section{4. $\mathcal{V}^{-}$-Palais-Smale SeQuences for the Functional $\mathcal{F}$}

In this section we study the Palais-Smale sequences for the functional $\mathcal{F}$ in the strip $\mathcal{F}^{-1}\left(\left[\delta_{0}^{2} / \mathrm{K}_{0}^{2}, \mathrm{M}_{0}^{2}\right]\right)$.

Definition 4.1. We say that $\left(x_{n}\right)_{n} \subset \mathfrak{M}$ is a $\mathcal{V}^{-}$-Palais-Smale sequence for $\mathcal{F}$ in the strip $\mathcal{F}^{-1}\left(\left[\delta_{0}^{2} / K_{0}^{2}, M_{0}^{2}\right]\right)$, if

$$
\frac{\delta_{0}^{2}}{\mathrm{~K}_{0}^{2}} \leqslant \mathcal{F}\left(x_{\mathrm{n}}\right) \leqslant M_{0}^{2}, \quad \forall \mathrm{n}
$$


and if for all (sufficiently large) $n \in \mathbb{N}$ and for all $V_{n} \in \mathcal{V}^{-}\left(x_{n}\right)$ such that $\left\|V_{n}\right\|_{*}=1$, the following holds:

$$
\mathrm{d} \mathcal{F}\left(\mathrm{x}_{n}\right)\left[\mathrm{V}_{\mathrm{n}}\right] \geqslant-\epsilon_{\mathrm{n}}
$$

where $\epsilon_{\mathrm{n}} \rightarrow 0^{+}$.

Note that, since $\bar{\Omega}$ is compact, if $\left(x_{n}\right)_{n} \subset \mathfrak{M}$ is a sequence such that $\mathcal{F}\left(x_{n}\right)$ is bounded, then $\left(x_{n}\right)$ admits a subsequence which is weakly convergent in $\mathrm{H}^{1}$ (and therefore uniformly convergent). Let us show that the weak limit $x$ of a $\mathcal{V}^{-}$-Palais-Smale sequence $\left(x_{n}\right)_{n}$ in the strip $\mathcal{F}^{-1}\left(\left[\delta_{0}^{2} / \mathrm{K}_{0}^{2}, M_{0}^{2}\right]\right)$, is in fact a strong limit, and it is a critical point of $\mathcal{F}$.

Proposition 4.2. Let $\left(x_{n}\right)_{n}$ be a $\mathcal{V}^{-}$-Palais-Smale sequence which is weakly convergent to a curve $\mathrm{x}$. Then $\left(\mathrm{x}_{\mathrm{n}}\right)_{\mathrm{n}}$ is strongly $\mathrm{H}^{1}$-convergent to $\mathrm{x}$.

Proof. Let us consider an auxiliary Riemannian metric $\bar{g}$ in $\bar{\Omega}$ for which the boundary $\partial \Omega$ is totally geodesic ${ }^{1}$, and let $\overline{\exp }$ denote the relative exponential map. Note that this means

$$
\text { for all } p \in \partial \Omega, \overline{\exp }_{p}^{-1}(\partial \Omega)=T_{p}(\partial \Omega) \text {. }
$$

For all $n$ sufficiently large, define the following vector field $V_{n}$ along the curve $x_{n}$ :

$$
V_{n}(s)=\left(\overline{\exp }_{x_{n}(s)}\right)^{-1}(x(s)) \text {. }
$$

This is well defined for $n$ sufficiently large, because $x_{n}$ tends to $x$ uniformly. Also, $\left\|V_{n}\right\|_{*}$ is bounded, because $\left(x_{n}\right)$ is bounded in $H^{1}$. It is also easy to check that, using the fact that $\partial \Omega$ is totally geodesic relatively to $\bar{g}, V_{n} \in \mathcal{V}^{-}\left(x_{n}\right)$ for all $n$. Namely, if $x_{n}(s) \in \partial \Omega$, since $x(s) \in \bar{\Omega}$, then $V_{n}(s)$ points inside $\Omega$. Similarly, for $s=0,1$, both $x_{n}(s)$ and $x(s)$ are in $\partial \Omega$, and thus $V(s)$ is tangent to $\partial \Omega$. Since $\left(x_{n}\right)$ is a $\mathcal{V}^{-}$-Palais-Smale sequence, by (4.2) we have:

$$
\liminf _{n \rightarrow \infty} \int_{0}^{1} g\left(\dot{x}_{n}, \frac{D}{d s} V_{n}\right) d s \geqslant 0
$$

Let $\mathrm{U}_{1}, \ldots, \mathrm{U}_{v}$ be the domains of local charts covering $\times([0,1])$, and let $\left[a_{i}, b_{i}\right](i=$ $1, \ldots, k)$ intervals covering $[0,1]$ such that $x\left(\left[a_{i}, b_{i}\right]\right) \subset U_{i}$ for all $i$. Using the fact that $V_{n}$ tends to 0 uniformly, $x_{n}$ tends to $x$ uniformly as $n \rightarrow \infty$, and that $\left\|\dot{x}_{n}\right\|_{L^{2}}$ is bounded, one sees easily that, using the above local charts, in any interval $\left[a_{i}, b_{i}\right]$ the covariant derivative $\frac{\mathrm{D}}{\mathrm{ds}} \mathrm{V}_{\mathrm{n}}$ is given by an expression of the form:

$$
\frac{\mathrm{D}}{\mathrm{d} s} \mathrm{~V}_{\mathrm{n}}=\dot{\mathrm{x}}-\dot{\chi}_{\mathrm{n}}+w_{\mathrm{n}}^{\mathrm{i}}
$$

where $w_{n}^{i}$ is $L^{2}$-convergent to 0 . Moreover, by the weak $L^{2}$-convergence of $\dot{x}_{n}$ to $\dot{x}$, we have:

$$
\int_{a_{i}}^{b_{i}} g\left(\dot{x}, \dot{x}-\dot{x}_{n}\right) d s \rightarrow 0 .
$$

Then, from (4.4) and (4.5) one obtains the $H^{1}$-convergence of $x_{n}$ to $x_{\text {. }}$

In particular, the strong $\mathrm{H}^{1}$-convergence implies that, if $x$ is the limit of a $\mathcal{V}^{-}$-PalaisSmale sequence for $\mathcal{F}$ in the strip $\mathcal{F}^{-1}\left(\left[\delta_{0}^{2} / \mathrm{K}_{0}^{2}, M_{0}^{2}\right]\right)$, then $\mathcal{F}(x) \geqslant \delta_{0}^{2} / 2 M_{0}^{2}$, and therefore $x$ is not a constant curve.

\footnotetext{
${ }^{1}$ There is a standard construction of metrics for which a given closed embedded submanifold of a differentiable manifold is totally geodesic. Such metrics are constructed in a tubular neighborhood first, using a normal bundle construction, and then extended using a partition of unity argument.
} 
Our next step will be the proof of existence of local vector fields in $\mathfrak{M}$, having the property that $\mathcal{F}$ is decreasing along their flow lines,. We will use the following notation: for all $\chi \in \mathfrak{M}$ and all $\rho>0$ set

$$
\mathrm{B}(x, \rho)=\left\{z \in \mathfrak{M}:\|z-x\|_{*}<\rho\right\}
$$

and

$$
\mathcal{U}_{x}(\rho)=B(x, \rho) \bigcup B(\mathcal{R} x, \rho),
$$

which is clearly $\mathcal{R}$-invariant. Let us also use the following terminology: let $\chi \in \mathfrak{M}$ and $\mu>0$ be fixed. We say that $\mathcal{F}$ has steepness greater than or equal to $\mu$ at $x$ if there exists $V_{x} \in \mathcal{V}^{-}(x)$ with:

(a) $\left\|V_{x}\right\|_{*}=1$,

(b) $\int_{0}^{1} g\left(\dot{x}, \frac{D}{d s} V_{x}\right) d s \leqslant-\mu$.

In this situation, $\mathrm{V}_{\mathrm{x}}$ is a direction of $\mu$-steep descent for $\mathcal{F}$ at $\mathrm{x}$.

For all $\delta>0$, we also define

$$
\mathcal{V}_{\delta}^{-}(x)=\left\{\mathrm{V} \in \mathcal{V}^{-}(x): g(\nabla \phi(x(s)), V(s)) \leqslant 0 \text { when } \phi(x(s)) \in[-\delta, \delta]\right\} .
$$

We can now prove the following:

Proposition 4.3. Let $x \in \mathfrak{M}$ be such that $\mathcal{F}(x) \in\left[\delta_{0}^{2} / \mathrm{K}_{0}^{2}, M_{0}^{2}\right]$ and let $\mu>0$ be fixed; assume that $\mathcal{F}$ has steepness greater than or equal to $\mu$ at $x$. Then, for any $\varepsilon>0$ there exist $\rho_{x}=\rho_{x}(\varepsilon)>0, \delta_{x}=\delta_{x}(\epsilon)>0$ and a $C^{1}$-vector field $\mathrm{V}=\mathrm{V}_{x, \epsilon}$ defined in $U_{x}\left(\rho_{x}\right)$, such that:

(i) $\mathrm{V}(\mathcal{R} z)=\mathcal{R} V(z)$;

(ii) $V(z) \in \mathcal{V}_{\delta_{x}}^{-}(z)$;

(iii) $\|\mathrm{V}(z)\|_{*}=1$;

(iv) $\int_{0}^{1} \mathrm{~g}\left(\dot{z}, \frac{\mathrm{D}}{\mathrm{ds}} \mathrm{V}(z)\right) \mathrm{d} s \leqslant-\mu+\varepsilon$,

for all $z \in \mathcal{U}_{x}\left(\rho_{x}\right)$.

Proof. Assume first $x \neq \mathcal{R} x$. Let $\mathrm{V}_{x}$ be a direction of $\mu$-steep descent for $\mathcal{F}$ at $x$.

Let us denote by $\overline{\exp }$ the exponential map of a smooth metric that makes $\partial \Omega$ totally geodesic. For $\rho>0$ sufficiently small and $z \in B(x, \rho)$, set:

$$
W(z)(s)=\left(d \overline{\exp }_{z(s)}(w(s))\right)^{-1}\left(V_{x}(s)\right)
$$

where $w(s)$ is defined by the relation:

$$
\overline{\exp }_{z(s)}(\mathcal{w}(s))=\chi(s), \quad \forall s .
$$

From (4.3) it follows:

$$
g(W(z)(0), \nabla \phi(z(0)))=g(W(z)(1), \nabla \phi(z(1)))=0 .
$$

Let $v$ the unit vector field in $\phi^{-1}\left(\left[-\delta_{0}, \delta_{0}\right]\right)$ defined by:

$$
v(y)=\frac{\nabla \phi(y)}{\sqrt{g(\nabla \phi(y), \nabla \phi(y))}} ;
$$

for $\sigma \in \mathbb{R}$ denote by $\sigma^{+}=\max \{\sigma, 0\}$ :

$$
\sigma^{+}=\frac{1}{2}(|s|+s)
$$


and define

$$
\begin{aligned}
\lambda=\lambda(\rho, \delta)=\sup \left\{g(W(z)(s), v(z(s)))^{+}\right. & \\
& s \in[0,1], z \in B(x, \rho), \phi(z(s)) \in[-\delta, \delta]\} .
\end{aligned}
$$

Note that

$$
\lim _{(\rho, \delta) \rightarrow(0,0)} \lambda(\rho, \delta)=0 .
$$

Then, for $\rho$ and $\delta$ sufficiently small, one has $\lambda \in\left[0, \frac{1}{2}[\right.$, and we can define

$$
a_{\lambda}=g(W(z)(\lambda), v(x(\lambda)))^{+}, \quad b_{\lambda}=g(W(z)(1-\lambda), v(x(1-\lambda)))^{+}
$$

which satisfy $0 \leqslant a_{\lambda}, b_{\lambda} \leqslant \lambda$, and therefore

$$
a_{\lambda} \rightarrow 0, \quad b_{\lambda} \longrightarrow 0 \quad \text { as } \quad(\rho, \delta) \longrightarrow(0,0) .
$$

Using $a_{\lambda}$ and $b_{\lambda}$, we now define the map $\chi_{\lambda}:[0,1] \rightarrow \mathbb{R}$ by:

$$
\chi_{\lambda}(s)= \begin{cases}g\left(W(z)(s), v(z(s))^{+},\right. & \text {if } s \in[0, \lambda] ; \\ \frac{b_{\lambda}-a_{\lambda}}{1-2 \lambda}(s-\lambda)+a_{\lambda}, & \text { if } s \in[\lambda, 1-\lambda] ; \\ g\left(W(z)(s), v(z(s))^{+},\right. & \text {if } s \in[1-\lambda, 1] .\end{cases}
$$

Now, let us consider piecewise linear maps $\theta, c: \mathbb{R} \rightarrow[0,1]$ satisfying:

$$
\left.\theta \equiv 0 \text { on }]-\infty,-\delta_{0}\right] \bigcup\left[\delta_{0},+\infty\left[\text { and } \theta \equiv 1 \text { on }\left[-\frac{\delta_{0}}{2}, \frac{\delta_{0}}{2}\right] .\right.\right.
$$

and

Finally, let us define

$$
c(s)= \begin{cases}s, & \text { if } s \in\left[0, \frac{1}{2}\right] \\ 1-s, & \text { if } s \in\left[\frac{1}{2}, 1\right]\end{cases}
$$

$$
W_{\lambda}(z)(s)=W(z)(s)-\left(\chi_{\lambda}(s)+\lambda c(s)\right) \theta(\phi(z(s)) v(z(s)) .
$$

We have

$$
\mathrm{g}\left(\mathrm{W}_{\lambda}(z)(\mathrm{s}), v(z(\mathrm{~s})) \leqslant 0 \text { for all } z \in \mathrm{B}(x, \rho) \text {, and for all s such that } \phi(z(s)) \in[-\delta, \delta]\right. \text {, }
$$

as we can see considering separately the cases $s \in[0, \lambda] \cup[1-\lambda, 1]$ and $s \in[\lambda, 1-\lambda]$. Moreover:

$$
\mathrm{g}\left(\mathrm{W}_{\lambda}(z)(0), v(z(0))=\mathrm{g}\left(\mathrm{W}_{\lambda}(z)(1), v(z(1))=0,\right.\right.
$$

which says that $W_{\lambda}(z) \in \mathcal{V}_{\delta}^{-}(z)$ for all $z \in \mathrm{B}(x, \rho)$. It is also easy to see that

$$
\lim _{(\rho, \delta) \rightarrow(0,0)} \sup _{z \in B(x, \rho)}\left\|W_{\lambda}(z)-V_{x}\right\|_{*}=0
$$

Since for all $\rho, \delta$ sufficiently small $W_{\lambda}(z) \not \equiv 0$, we can define

$$
v_{\lambda}(z)(s)=\frac{W_{\lambda}(z)(s)}{\left\|W_{\lambda}(z)\right\|_{*}} .
$$

But $\left\|V_{x}\right\|_{*}=1$, so by (4.12):

$$
\lim _{(\rho, \delta) \rightarrow(0,0)} \sup _{z \in B(x, \rho)}\left\|V_{\lambda}(z)-V_{x}\right\|_{*}=0,
$$

and we get property (iv). 
Now, recalling that we are assuming $x \neq \mathcal{R} x$, we extend $V_{\lambda}$ to $\mathcal{R}\left(B\left(x, \rho_{\lambda}\right)\right)$ by setting:

$$
V_{\lambda}(\mathcal{R} z)=\mathcal{R} V_{\lambda}(z)
$$

Then, the desired vector field $V$ is obtained by setting $V=V_{\lambda}$, with $\lambda=\lambda(\rho, \delta), \rho=\rho(\varepsilon)$, and $\delta=\delta(\varepsilon)$ choosen sufficiently small (depending on $\varepsilon$ ).

Now, observe that if $x=\mathcal{R} x$ and $V_{x}$ is a direction of $\mu$-steep descent we can consider

$$
\widetilde{V}_{x}=\frac{V_{x}+\mathcal{R} V_{x}}{\left\|V_{x}+\mathcal{R} V_{x}\right\|_{*}}
$$

Since

$$
\int_{0}^{1} \mathrm{~g}\left(\dot{x}, \frac{\mathrm{DV}}{\mathrm{d} s}\right) \mathrm{ds}=\int_{0}^{1} \mathrm{~g}\left(\dot{x}, \frac{\mathrm{D}}{\mathrm{d} s} \mathcal{R} V_{x}\right) \mathrm{ds}
$$

we see that $V_{x}+\mathcal{R} V_{x} \not \equiv 0$, and $\widetilde{V}_{x}$ is a direction of $\mu$-steep descent for $\mathcal{F}$. We can therefore assume that the direction $V_{x}$ of $\mu$-steep descent for $\mathcal{F}$ at $x$ is such that

$$
V_{x}=\mathcal{R} V_{x} \text {. }
$$

If $W_{\lambda}(z)$ is the vector field as in (4.11), we can finally choose

$$
\mathrm{V}(z)(s)=\frac{W_{\lambda}(z)(s)+W_{\lambda}(\mathcal{R} z)(s)}{\left\|W_{\lambda}(z)+W_{\lambda}(\mathcal{R} z)(s)\right\|_{*}}
$$

\section{Deformation Lemmas}

5.1. Admissible homotopies. Let $\mathfrak{C}_{0}$ be as in (2.17). We will consider continuous maps $h:[0,1] \times \mathfrak{M} \rightarrow \mathfrak{M}$ satisfying the following properties:

$$
\begin{gathered}
h(0, \cdot) \text { is the identity map in } \mathfrak{M} ; \\
h(\tau, \gamma)=\gamma \text { for all } \tau \in[0,1] \text {, for all } \gamma \in \mathfrak{C}_{0} ;
\end{gathered}
$$

(5.3) $h(\tau, \cdot)$ is $\mathcal{R}$-equivariant, i.e. $h(\tau, \mathcal{R} \gamma)=\mathcal{R h}(\tau, \gamma)$ for all $\tau \in[0,1], \gamma \in \mathfrak{M}$.

We will call admissible homotopies all maps $h:[0,1] \times \mathcal{D} \rightarrow \mathfrak{M}$ satisfying (5.1)-(5.3), and we will denote by $\mathcal{H}$ the set of admissible homotopies.

5.2. Admissible homotopies and weak slope. Denote by $d$ the distance (2.7). Denote by $\mathcal{H}$ the class of all continuous homotopies $h$ defined at the above section. Let $\mathcal{U}_{x}(\rho)$ be as in (4.6).

According to $[3,7]$ we give the following definitions

Definition 5.1. For every $x \in \mathfrak{M}$ we denote by $|d \mathcal{F}|(x)$ the supremum of the $\sigma$ 's in $[0,+\infty[$ such that there exists $\delta>0$ and a continuous homotopy $h \in \mathcal{H}$ such that

$$
\begin{gathered}
d(h(\tau, z), z) \leqslant \tau, \text { for every } z \in \mathcal{U}_{x}(\delta), \text { and } \tau \in[0, \delta], \\
\mathcal{F}(h(\tau, z)) \leqslant \mathcal{F}(z)-\sigma \tau \text {, for every } z \in \mathcal{U}_{x}(\delta) \text { and } \tau \in[0, \delta] .
\end{gathered}
$$

The extended real number $|\mathrm{d} \mathcal{F}|(x)$ is called weak slope of $\mathcal{F}$ at $x$ (with respect to $\mathcal{H}$ ).

Definition 5.2. A curve $x \in \mathfrak{M}$ is called a critical curve for $\mathcal{F}$ if $|\mathrm{d} \mathcal{F}|(x)=0$. A real number $c$ is called critical value for $\mathcal{F}$ if there exists $x \in \mathfrak{M}$ critical curve such that $\mathcal{F}(x)=$ c.

Definition 5.3. We say that $\left(x_{\mathfrak{n}}\right)_{\mathfrak{n}} \subset \mathfrak{M}$ is a Palais-Smale sequence for $\mathcal{F}$ in the strip $\mathcal{F}^{-1}\left(\left[\delta_{0}^{2} / \mathrm{K}_{0}^{2}, M_{0}^{2}\right]\right)$, if $x_{n}$ satisfies (4.1) and

$$
|\mathrm{d} \mathcal{F}|\left(x_{n}\right) \longrightarrow 0, \quad \text { as } n \rightarrow \infty .
$$


Remark 5.4. As observed in [3], it is an obvious consequence of its definition that the function $|\mathrm{d} \mathcal{F}|: \mathfrak{M} \rightarrow[0,+\infty]$ is lower semi-continuous. It follows that if a Palais-Smale sequence $x_{n}$ converges to $x$, the curve $x$ is a critical curve.

Now, using the integral flow of the $C^{1}$-vector field $V_{x, \rho_{x}}$ given in Proposition 4.3, we can easily adapt to our setting the the proof of [3, Theorem 1.1.2] to obtain the following:

Proposition 5.5. For every $x \in \mathfrak{M}$, the following inequality holds:

$$
\sup \left\{-\mathrm{d} \mathcal{F}(x)[\mathrm{V}]: \mathrm{V} \in \mathcal{V}^{-}(\mathrm{x}),\|\mathrm{V}\|_{*}=1\right\} \leqslant|\mathrm{d} \mathcal{F}|(x) .
$$

Corollary 5.6. If $x$ is a critical curve for $\mathcal{F}$, then $x$ is a $\mathcal{V}^{-}$-critical curve. Moreover, if $\left(x_{n}\right)$ is a Palais-Smale sequence for $\mathcal{F}$, then it is also a $\mathcal{V}^{-}$-Palais-Smale sequence, therefore it is strongly convergent (cf Proposition 4.2).

For $a \in \mathbb{R}$, denote by $\mathcal{F}^{a}$ the closed a-sublevel of $\mathcal{F}$ :

$$
\mathcal{F}^{\mathrm{a}}=\{x \in \mathfrak{M}: \mathcal{F}(x) \leqslant \mathrm{a}\} .
$$

Using the deformation results proved in $[3,4]$, we now have:

Proposition 5.7. Suppose that $\mathrm{c} \in\left[\delta_{0}^{2} / \mathrm{K}_{0}^{2}, \mathrm{M}_{0}^{2}\right]$ is not a critical value for $\mathcal{F}$. Then, there exists $\varepsilon=\varepsilon(\mathrm{c})>0$ and a homotopy $\eta \in \mathcal{H}$ such that

$$
\eta\left(1, \mathcal{F}^{\mathrm{c}+\epsilon}\right) \subset \mathcal{F}^{\mathrm{c}-\epsilon} .
$$

5.3. Two elementary lemmas on homotopies on the sphere. In preparation for the final result of this section, we give a statement and a short proof of two auxiliary results concerning homotopies on spheres. Let $\mathrm{g}$ be a Riemannian metric on $\mathbb{S}^{\mathrm{m}},(\mathrm{m}=\mathrm{N}-1$ for our applications), and let $\delta_{g}>0$ be the minimum of the injectivity radius function of $\left(\mathbb{S}^{m}, g\right)$. Here, dist will denote the distance function on $\mathbb{S}^{m}$ induced by $g$.

Lemma 5.8. Let $\mathcal{C} \subset \mathbb{S}^{m} \times \mathbb{S}^{m}$ be a closed set such that:

$$
0<\alpha \leqslant \min _{(A, B) \in \mathcal{C}} \operatorname{dist}(A, B)<\delta_{g} .
$$

There exists a continuous map $\mathfrak{s}^{\mathfrak{e}}:\left[0, \delta_{g}-\alpha\right] \times \mathfrak{C} \rightarrow \mathbb{S}^{\mathrm{m}}$, such that, denoting $\mathfrak{s}^{\mathfrak{e}}(\tau, \cdot)=$ $\mathfrak{s}_{\tau}^{\mathfrak{e}}$ :

(1) $\mathfrak{s}_{0}^{\mathfrak{e}}(A, B)=B$ for all $(A, B) \in \mathcal{C}$;

(2) $\tau \mapsto \operatorname{dist}\left(A, \mathfrak{s}_{\tau}^{\mathcal{e}}(A, B)\right)$ is nondecreasing for all $(A, B) \in \mathcal{C}$;

(3) $\mathfrak{s}_{\tau}^{\mathcal{C}}(A, B)=B$ for all $(A, B) \in \mathcal{C}$ with $\operatorname{dist}(A, B) \geqslant \delta_{g}$;

(4) $\min _{(A, B) \in \mathcal{C}} \operatorname{dist}\left(A, \mathfrak{s}_{\delta_{g}-\alpha}^{\mathcal{C}}(A, B)\right) \geqslant \delta_{g}$, for all $(A, B) \in \mathcal{C}$.

Proof. For all $(A, B) \in \mathcal{C}$ with $\operatorname{dist}(A, B)<\delta_{g}$, denote by $\gamma_{A, B}$ the minimal unit speed g-geodesic in $\mathbb{S}^{m}$ from $B$ to $A$, and with $\gamma_{A, B}(0)=B$. For $(A, B) \in \mathcal{C}$, one can define:

$$
\mathfrak{s}_{\tau}^{\mathcal{C}}(A, B)= \begin{cases}B, & \text { if } \operatorname{dist}(A, B) \geqslant \delta_{g} ; \\ \gamma_{A, B}\left(\tau \cdot \frac{\operatorname{dist}(A, B)-\delta_{g}}{\delta_{g}-\alpha}\right), & \text { if } \operatorname{dist}(A, B)<\delta_{g} .\end{cases}
$$

The continuity of $\mathfrak{s}^{\mathfrak{C}}$ and properties (1)-(4) are easily verified.

The continuous map $\left[0, \delta_{g}-\alpha\right] \times \mathcal{C} \ni(\tau,(A, B)) \mapsto\left(A, \mathfrak{s}_{\tau}^{\mathcal{C}}(A, B)\right) \in \mathbb{S}^{m} \times \mathbb{S}^{m}$ will be called the endpoint separating homotopy of the set $\mathcal{C}$. 
Lemma 5.9. Let $\mathcal{C} \subset \mathbb{S}^{m} \times \mathbb{S}^{m}$ be a closed set, and let $\mathrm{h}_{1}:[0,1] \times \mathcal{C} \rightarrow \mathbb{S}^{m}$ be a continuous map. There exists $\tau_{0}>0$ such that, for all $(\mathrm{A}, \mathrm{B}) \in \mathcal{C}$ and all $\mathrm{E} \in \mathbb{S}^{\mathrm{m}}$ for which:

$$
\operatorname{dist}\left(h_{1}(a,(A, B)), E\right) \geqslant \delta_{g}, \quad \text { and } \operatorname{dist}\left(h_{1}(b,(A, B)), E\right) \leqslant \frac{1}{2} \delta_{g},
$$

for some $0 \leqslant \mathrm{a}<\mathrm{b} \leqslant 1$, then $\mathrm{b}-\mathrm{a} \geqslant \tau_{0}$.

Proof. It follows immediately from the uniform continuity of $h_{1}$ and a compactness argument.

5.4. The deformation lemma for critical levels. Assume that the number of OGC's is finite (otherwise our main result does not need a proof!), and let us denote them by $\gamma_{1}, \ldots, \gamma_{k}$. Fix $r_{*}>0$ such that

- the sets $\left\{y \in \partial \Omega: \operatorname{dist}\left(y, \gamma_{i}(0)\right)<r_{*}\right\}$ and $\left\{y \in \partial \Omega: \operatorname{dist}\left(y, \gamma_{i}(1)\right)<r_{*}\right\}$ are contractible in $\Omega$ for all $i$ and pairwise disjoint;

- $B\left(\gamma_{i}, r_{*}\right) \cap B\left(\gamma_{j}, r_{*}\right)=\emptyset$ for all $i \neq j$;

- $\mathrm{B}\left(\gamma_{i}, r_{*}\right) \cap \mathrm{B}\left(\mathcal{R} \gamma_{i}, r_{*}\right)=\emptyset$ for all $i$ (note that $\gamma_{i} \neq \mathcal{R} \gamma_{i}$ for all $i$ ).

For every $\left.r \in] 0, r_{*}\right]$ set

$$
\mathcal{O}_{\mathrm{r}}=\bigcup_{i=1}^{k} \mathcal{u}_{\gamma_{i}}(\mathrm{r}) \text {, }
$$

(cf (4.6)). As in Proposition 5.7, using the weak slope theory we have:

Proposition 5.10. Let $\mathrm{c} \in\left[\delta_{0}^{2} / \mathrm{k}_{0}^{2}, \mathrm{M}_{0}^{2}\right]$ be a critical value for $\mathcal{F}$. Then there exists $\varepsilon\left(\mathrm{r}_{*}\right)>0$ and a homotopy $\eta \in \mathcal{H}$ such that

$$
\eta\left(1, \mathcal{F}^{\mathrm{c}+\epsilon\left(\mathrm{r}_{*}\right)} \backslash \mathcal{O}_{\mathrm{r}_{*}}\right) \subset \mathcal{F}^{\mathrm{c}-\epsilon\left(\mathrm{r}_{*}\right)} .
$$

Now let $\mathfrak{D}$ denote the family of all closed $\mathcal{R}$-invariant subset of $\mathfrak{C}$. Given $\mathcal{D} \in \mathfrak{D}$ and $\mathrm{h} \in \mathcal{H}$, we denote by $\mathcal{A}_{\mathrm{h}}$ the $\mathcal{R}$-invariant set:

$$
\mathcal{A}_{\mathrm{h}}=\left\{x \in \mathcal{D}: \mathrm{h}(1, x) \in \mathcal{O}_{\mathrm{r}_{*}}\right\} .
$$

For the minimax theory we will need the following:

Proposition 5.11. Fix $\mathcal{D} \in \mathfrak{D}, \mathrm{h} \in \mathcal{H}$, and let $\mathcal{A}_{\mathrm{h}}$ be as in (5.8). Then, there exists a continuous map

$$
k_{*}:[0,1] \times \mathcal{A}_{\mathrm{h}} \longrightarrow \mathfrak{C} \backslash \mathfrak{C}_{0}
$$

such that:

(a) $k_{*}(0, x)=x$ for every $x \in \mathcal{A}_{h}$;

(b) $k_{*}(\tau, \mathcal{R} x)=\mathcal{R k}_{*}(\tau, x)$ for all $\tau \in[0,1]$, for all $x \in \mathcal{A}_{h}$;

(c) $k_{*}\left(1, \mathcal{A}_{h}\right)=\left\{y_{0}, R y_{0}\right\}$ for some $y_{0} \in \mathfrak{C} \backslash \mathfrak{C}_{0}$.

Proof. The first observation is that a homotopy that satisfies (a), (b) and (c) above, but taking values in $\mathfrak{C}$ (i.e., possibly having points of $\mathfrak{C}_{0}$ in its image), does exist. This follows from the fact that, by definition, the homotopy $h$ carries $\mathcal{A}_{h}$ into the set $\mathcal{O}_{r_{*}}$. For $\mathrm{r}_{*}>0$ small enough, this set is retractible in $\mathfrak{M}$ onto the finite set $\left\{\gamma_{1}, \ldots, \gamma_{k}, \mathcal{R} \gamma_{1}, \ldots, \mathcal{R} \gamma_{k}\right\}$, and again this finite set is retractible in $\mathfrak{M}$, say, to the two-point set $\left\{\gamma_{1}, \mathcal{R} \gamma_{1}\right\}$. Composing with the endpoints mapping, $\mathfrak{M} \ni \gamma \mapsto(\gamma(0), \gamma(1)) \in \partial \Omega \times \partial \Omega \cong \mathfrak{C}$, one obtains a homotopy $h_{*}:[0,1] \times \mathcal{A}_{h} \rightarrow \mathfrak{C}$ that carries $\mathcal{A}_{\mathrm{h}}$ to the two-point set $\left\{y_{0}, \mathcal{R} y_{0}\right\}$, where $y_{0}=\left(\gamma_{1}(0), \gamma_{1}(1)\right)$. 
Let us show how to use $h_{*}$ to construct another homotopy carrying $\mathcal{A}_{\mathrm{h}}$ to the two-point set $\left\{y_{0}, \mathcal{R} y_{0}\right\}$ in $\mathfrak{C} \backslash \mathfrak{C}_{0}$. Note that $\mathcal{A}_{h} \cap \mathfrak{C}_{0}=\emptyset$; namely, $h(\tau, \cdot)$ fixes all points of $\mathfrak{C}_{0}$ for all $\tau \in[0,1]$ (by definition of admissible homotopies), and $\mathcal{O}_{\mathrm{r}_{*}} \cap \mathfrak{C}_{0}=\emptyset$ for $\mathrm{r}_{*}>0$ small enough.

The second observation is that $\mathcal{A}_{\mathrm{h}}$ can be written as the disjoint union $\mathrm{F}_{1} \cup \mathrm{F}_{2}$ of two closed sets $\mathrm{F}_{1}, \mathrm{~F}_{2} \subset \mathcal{D}$, with $\mathcal{R F}_{1}=\mathrm{F}_{2}$; namely:

$$
\mathrm{F}_{1}=\mathrm{h}_{*}(1, \cdot)^{-1}\left(\mathrm{y}_{0}\right), \quad \text { and } \quad \mathrm{F}_{2}=\mathrm{h}_{*}(1, \cdot)^{-1}\left(\mathcal{R} \mathrm{y}_{0}\right) .
$$

To conclude the proof, it suffices to show that $F_{1}$ is contractible in $\mathfrak{C}_{\mathfrak{c}} \backslash \mathfrak{C}_{0}$ to the singleton $\left\{y_{0}\right\}$ via some homotopy $\widetilde{h}_{*}:[0,1] \times F_{1} \rightarrow \mathfrak{C} \backslash \mathfrak{C}_{0}$; the desired homotopy $k_{*}$ will then be obtained by extending $\widetilde{h}_{*}$ to $[0,1] \times F_{2}$ by $\mathcal{R}$-equivariance. The map $\widetilde{h}_{*}$ will be constructed in two stages. First, we will find a continuous map $\ell:[0,1] \times F_{1} \rightarrow \mathfrak{C} \backslash \mathfrak{C}_{0}$, with $\ell(\tau,(A, B))=\left(\ell_{\tau}^{1}(A, B), \ell_{\tau}^{2}(A, B)\right)$ for all $(A, B) \in F_{1}$, such that, denoting by $\delta_{g}>0$ the minimum of the injectivity radius function of the metric $\mathrm{g}$ in $\partial \Omega \cong \mathbb{S}^{\mathrm{N}}$ :

(i) $\ell(0,(A, B))=(A, B)$, for all $(A, B) \in F_{1}$;

(ii) $\min _{\substack{\tau \in[0,1] \\(A, B) \in F_{1}}} \operatorname{dist}\left(\ell_{\tau}^{1}(A, B), \ell_{\tau}^{2}(A, B)\right) \geqslant \frac{1}{2} \delta_{g}$;

(iii) $\ell_{1}^{1}\left(F_{1}\right)$ is the singleton $\left\{\gamma_{1}(0)\right\}$.

From (i) and (ii) it follows that the set $\ell_{1}^{2}\left(F_{1}\right)$ is contained in the complement $W$ of a disk of radius $\frac{1}{2} \delta_{g}$ centered at $\gamma(0)$ in $\mathbb{S}^{N}$. This set $W$ is contractible to the singleton $\{\gamma(1)\}$ in $\mathbb{S}^{n}$ through a homotopy that fixes all points in a disk of radius $\frac{1}{4} \delta_{g}$ centered at $\gamma(0)$. The map $\widetilde{h}_{*}$ will be obtained by applying $\ell$ first, and then concatenating such homotopy that contracts $W$ to $\{\gamma(1)\}$.

Thus, we are left with the construction of the map $\ell:[0,1] \times F_{1} \rightarrow \mathfrak{C} \backslash \mathfrak{C}_{0}$ as described above. To this aim, we consider the map $h_{*}:[0,1] \times F_{1} \rightarrow \mathfrak{C}$ that contracts $F_{1}$ to the point $y_{0} \in \mathfrak{C}$; let us write $h_{*}(\tau,(A, B))=\left(h_{\tau}^{1}(A, B), h_{\tau}^{2}(A, B)\right)$ for all $(A, B) \in F_{1}$ and $\tau \in[0,1]$. Note that

$$
h_{1}^{1}\left(F_{1}\right)=\left\{\gamma_{1}(0)\right\} .
$$

For our construction, we will only employ the first component $h^{1}$ of the homotopy $h_{*}$; we define $\bar{h}_{1}:[0,1] \times F_{1} \rightarrow \mathfrak{C} \cong \mathbb{S}^{n} \times \mathbb{S}^{n}$ by:

$$
\bar{h}_{1}(\tau,(A, B))=\left(h_{\tau}^{1}(A, B), B\right) .
$$

The desired homotopy $\ell$ will be obtained by concatenating successively stages of the homotopy $\bar{h}_{1}$ and the "endpoint separating homotopy" (Lemma 5.8), as we will describe below. It must be observed that $\bar{h}_{1}(\tau, \cdot)$ acts only on the first component of a point $(A, B) \in$ $F_{1}$, while the endpoint separating homotopy acts only on the second component of $(A, B)$.

Up to a first application of the endpoint-separating homotopy to the set $F_{1}$, we can assume:

$$
\min _{(A, B) \in F_{1}} \operatorname{dist}(A, B) \geqslant \delta_{g}
$$

Let $t_{1}>0$ be the smallest number in $\left.] 0,1\right]$ (or $t_{1}=1$ if no such number exists) such that $\operatorname{dist}\left(h_{t_{1}}^{1}(A, B), B\right) \leqslant \frac{1}{2} \delta_{g}$. On the interval $\left[0, t_{1}\right]$, the homotopy $\ell$ is defined to be equal to $\bar{h}_{1}$. If $t_{1}=1$, then our proof is concluded, by (5.9).

Assume $t_{1}<1$; after this instant, we apply again the endpoint-separating homotopy to the set:

$$
\left\{\left(h_{t_{1}}^{1}(A, B), B\right):(A, B) \in F_{1}\right\} ;
$$


which is then mapped to the set $\mathrm{F}_{1}^{\prime}$, that can be written as:

$$
F_{1}^{\prime}=\left\{\left(h_{t_{1}}^{1}(A, B), B_{1}(A, B)\right):(A, B) \in F_{1}\right\}
$$

for a suitable continuous map $B_{1}: F_{1} \rightarrow \mathfrak{C}$. This set now satisfies:

$$
\min _{\left(A_{1}, B_{1}\right) \in F_{1}^{\prime}} \operatorname{dist}\left(A_{1}, B_{1}\right) \geqslant \delta_{g}
$$

and we can apply again the homotopy $\bar{h}_{1}:\left[t_{1}, t_{2}\right] \times F_{1}^{\prime} \rightarrow \mathfrak{C}$, where $t_{2}$ is the smallest number in $\left.] t_{1}, 1\right]$ (or $t_{2}=1$ if no such number exists) such that $\operatorname{dist}\left(h_{t_{2}}^{1}(A, B), B_{1}(A, B)\right) \leqslant$ $\frac{1}{2} \delta_{g}$. If $t_{2}=1$, then our proof is concluded. Otherwise, we keep repeating successive applications of the endpoint separating homotopy followed by $\bar{h}_{1}$.

The conclusion is obtained by observing that our procedure terminates after a finite number of steps, that is, at some point we will be able to apply the homotopy $\bar{h}_{1}$ until the final instant $t=1$. This follows easily from the fact that, using the uniform continuity of $\bar{h}_{1}$, the difference $t_{k+1}-t_{k}$ of the instants defined in our construction must be bounded from below by some positive constant $\tau_{0}$, say $\tau_{0} \geqslant \frac{1}{n_{0}}$, for some $n_{0} \in \mathbb{N}$, see Lemma 5.9 for a precise statement. Thus, our procedure stops after at most $n_{0}$ steps.

\section{Proof of our MAin Theorem}

6.1. Relative Lusternik-Schinerlmann category. For the minimax theory we will use a suitable version of Lusternik-Schinerlmann relative category, as defined for instance in [9, Definition 3.1]. Other definitions of the relative category and relative cohomological indexes can be found e.g. in [8] and the references therein.

Definition 6.1. Let $X$ be a topological space and $y$ a closed subset of $X$. A closed subset $\mathrm{F}$ of $X$ has relative category equal to $\mathrm{k} \in \mathbb{N}(\operatorname{cat} X, y(\mathrm{~F})=\mathrm{k})$ if $\mathrm{k}$ is the minimal positive integer such that $F \subset \bigcup_{i=0}^{k} A_{i}$, where $\left\{A_{i}\right\}_{i=1}^{k}$ is a family of open subsets of $\mathcal{X}$ satisfying:

- $\mathrm{F} \cap \mathrm{Y} \subset \mathrm{A}_{0}$;

- for all $i=0, \ldots, k$ there exists $h_{i} \in C^{0}\left([0,1] \times A_{i}, X\right)$ with the following properties:

(1) $h_{i}(0, x)=x, \forall x \in A_{i}, \forall i=0, \ldots, k$;

(2) for any $i=1, \ldots, k$ :

(a) there exists $x_{i} \in X \backslash y$ such that $h_{i}\left(1, A_{i}\right)=\left\{x_{i}\right\}$;

(b) $h_{i}:\left([0,1] \times A_{i}\right) \subset X \backslash y$;

(3) if $i=0$ :

(a) $h_{0}\left(1, A_{0}\right) \subset y$;

(b) $h_{0}\left(\tau, A_{0} \cap y\right) \subset y, \forall \tau \in[0,1]$.

For any $\mathrm{X} \subset \mathfrak{M}$ which is $\mathcal{R}$-invariant, we denote by $\widetilde{X}$ the quotient space with respect to the equivalence relation induced by $\mathcal{R}$.

The topological invariant employed in our theory is the relative category cat $\widetilde{\mathfrak{C}}_{\mathfrak{\mathfrak { C }}, \widetilde{\mathfrak{C}}_{\mathfrak{o}}}\left(\widetilde{\mathfrak{C}}^{\mathfrak{C}}\right)$. In [11] it has been shown that

$$
\operatorname{cat}_{\widetilde{\mathfrak{C}}, \widetilde{\mathfrak{C}_{0}}}(\widetilde{\mathfrak{C}}) \geqslant N
$$

using the topological properties of the $(\mathrm{N}-1)$-dimensional real projective space. 
6.2. The minimax argument. Recall that $\mathfrak{D}$ denotes the class of all closed $\mathcal{R}$-invariant subsets of $\mathfrak{C}$ and that, for $\mathcal{D} \in \mathfrak{D}$, the symbol $\mathcal{H}$ denotes the set of admissible homotopies, see Section 5.1. Define, for every $i=1, \ldots, N$,

$$
\Gamma_{i}=\left\{\mathcal{D} \in \mathfrak{D}: \operatorname{cat}_{\widetilde{\mathfrak{C}}, \widetilde{\mathfrak{C}}_{\mathfrak{o}}}(\widetilde{\mathcal{D}}) \geqslant i\right\},
$$

and

$$
c_{i}=\inf _{\substack{\mathcal{D} \in \Gamma_{i}, h \in \mathcal{H}}} \sup \{\mathcal{F}(h(1, x)): x \in \mathcal{D}\} .
$$

Remark 6.2. Every $c_{i}$ is a real number. Indeed $\mathcal{F} \geqslant 0$ so $c_{i} \geqslant 0$ for all $i$. Moreover, the identity homotopy is in $\mathcal{H}$ and $\widetilde{\mathfrak{C}} \in \Gamma_{i}$ for any $i$, therefore by (2.18),

$$
c_{i} \leqslant M_{0}^{2}, \quad \text { for every } i \text {. }
$$

Given continuous maps $\eta_{1}:[0,1] \times \mathrm{F}_{1} \rightarrow \mathfrak{M}$ and $\eta_{2}:[0,1] \times \mathrm{F}_{2} \rightarrow \mathfrak{M}$ such that $\eta_{1}\left(1, F_{1}\right) \subset F_{2}$, we define the concatenation of $\eta_{1}$ and $\eta_{2}$ as the map $\eta_{2} \star \eta_{1}:[0,1] \times F_{1} \rightarrow$ $\mathfrak{M}$ given by

$$
\eta_{2} \star \eta_{1}(t, x)= \begin{cases}\eta_{1}(2 t, x), & \text { if } t \in\left[0, \frac{1}{2}\right], \\ \eta_{2}\left(2 t-1, \eta_{1}(1, x)\right), & \text { if } t \in\left[\frac{1}{2}, 1\right] .\end{cases}
$$

The following lemmas describe the properties of the $c_{i}$ 's.

Lemma 6.3. The following statements hold:
(1) $\mathrm{c}_{1} \geqslant \frac{\delta_{0}^{2}}{\mathrm{~K}_{0}^{2}}$
(2) $\mathrm{c}_{1} \leqslant \mathrm{c}_{2} \leqslant \cdots \leqslant \mathrm{c}_{\mathrm{N}}$.

Lemma 6.4. For all $i=1, \ldots, N, c_{i}$ is a critical value of $\mathcal{F}$.

Lemma 6.5. Assume that the number of OGC's in $\bar{\Omega}$ is finite. Then:

$$
c_{i}<c_{i+1} \text {. }
$$

for all $\mathrm{i}=1, \ldots, \mathrm{N}-1$.

Proof of Lemma 6.3. Let us prove (1). Assume by contradiction $c_{1}<\frac{\delta_{0}^{2}}{K_{0}^{2}}$, and fix $\varepsilon>0$ so that $c_{1}+\epsilon<\frac{\delta_{0}^{2}}{K_{0}^{2}}$. By (6.2) and (6.3) there exists $\mathcal{D}_{\varepsilon} \in \Gamma_{1}$, and $h_{\varepsilon} \in \mathcal{H}$ such that

$$
\mathcal{F}\left(h_{\varepsilon}(1, x)\right)<\frac{\delta_{0}^{2}}{K_{0}^{2}}, \quad \text { for any } x \in \mathcal{D}_{\varepsilon} .
$$

Then, by Corollary 2.2,

$$
\inf \left\{\phi\left(h_{\varepsilon}(1, x)(s)\right): s \in[0,1], x \in \mathcal{D}_{\varepsilon}\right\} \geqslant-\delta_{0} .
$$

Using the retraction $\mathbf{r}$ in (2.3), we can then construct a homotopy $\Pi$ that carries $h_{\varepsilon}\left(1, \mathcal{D}_{\varepsilon}\right)$ onto $\partial \Omega$. Finally, we define the homotopy

$$
H(\tau, x)(s)=x\left((1-\tau) s+\frac{\tau}{2}\right)
$$

such homotopy carries each curve $x$ to the constant curve $x(1 / 2)$. We apply such $H$ to the curves of $\left(\Pi \star h_{\varepsilon}\right)\left(1, \mathcal{D}_{\varepsilon}\right)$, obtaining that cat ${\widetilde{\mathfrak{C}}, \widetilde{\mathfrak{C}}_{0}}_{\tilde{\mathcal{D}}_{\varepsilon}}\left(\widetilde{\mathcal{D}}_{\varepsilon}\right)=0$, in contradiction with the definition of $\Gamma_{1}$. 
To prove (2), fix $i \in\{1, \ldots, N-1\}$; by (6.3), for every $\varepsilon>0$ there exists $\mathcal{D} \in \Gamma_{i+1}$ and $h \in \mathcal{H}$ such that

$$
\mathcal{F}(h(1, x)) \leqslant c_{i+1}+\varepsilon, \quad \forall x \in \mathcal{D} .
$$

Since $\Gamma_{i+1} \subset \Gamma_{i}$. by definition of $c_{i}$ we deduce $c_{i} \leqslant c_{i+1}+\varepsilon$, and (2) is proved, since $\varepsilon$ is arbitrary.

Proof of Lemma 6.4. Assume by contradiction that $c_{i}$ is not a critical value for some $i$. Take $\varepsilon=\varepsilon\left(c_{i}\right)$ as in Proposition 5.7, and choose $\mathcal{D}_{\varepsilon} \in \mathfrak{D}, h \in \mathcal{H}$ such that

$$
\mathcal{F}\left(h_{\epsilon}(1, x)\right) \leqslant c_{i}+\varepsilon, \quad \text { for all } x \in \mathcal{D}_{\varepsilon} .
$$

Now let $\eta$ be as in Proposition 5.7. Since $\eta \star h_{\varepsilon} \in \mathcal{H}$ and

$$
\mathcal{F}\left(\left(\eta \star h_{\varepsilon}\right)(1, x)\right) \leqslant c_{i}-\varepsilon, \quad \text { for all } x \in \mathcal{D}_{\varepsilon},
$$

we obtain a contradiction with (6.3).

Proof of Lemma 6.5. Assume by contradiction that (6.6) does not hold. Then there exists $i \in\{1, \ldots, N-1\}$ such that

$$
\mathrm{c}:=\mathrm{c}_{\mathrm{i}}=\mathrm{c}_{\mathrm{i}+1} .
$$

Take $\varepsilon_{*}=\varepsilon\left(\mathrm{r}_{*}\right)$ as in Proposition 5.10, $\mathcal{D}_{\mathrm{i}+1} \in \Gamma_{\mathrm{i}+1}$ and $\mathrm{h} \in \mathcal{H}$, such that

$$
\mathcal{F}(h(1, x)) \leqslant c+\varepsilon_{*}, \quad \text { for all } x \in \mathcal{D}_{i+1} .
$$

Let $\mathcal{A}_{h}$ be the open set defined in (5.8); by Proposition 5.11, its projection $\widetilde{\mathcal{A}_{h}}$ in the quotient space $\widetilde{\mathfrak{C}}$ is contractible in $\widetilde{\mathfrak{C}} \backslash \widetilde{\mathfrak{C}}_{0}$. Then, by definition of $\Gamma_{i}$, and using simple properties of the relative category, the closed set $\mathcal{D}_{i}:=\mathcal{D}_{i+1} \backslash \mathcal{A}_{h}$ belongs to $\Gamma_{i}$. Now, let $\eta$ be as in Proposition 5.10. We have

$$
\mathcal{F}((\eta \star h)(1, x)) \leqslant c-\varepsilon_{*}, \quad \text { for all } x \in \mathcal{D}_{i},
$$

in contradiction with the definition of $\Gamma_{i}$. This concludes the proof.

6.3. Proof of the main theorem. Using Lemmas 6.3-6.5 it will be sufficient to prove that if $x_{1}$ and $x_{2}$ are OGC's:

$$
x_{1}([0,1])=x_{2}([0,1]) \quad \Longrightarrow \quad \mathcal{F}\left(x_{1}\right)=\mathcal{F}\left(x_{2}\right) .
$$

Since $\dot{x}_{1}$ and $\dot{x}_{2}$ are never vanishing, if $x_{1}([0,1])=x_{2}([0,1])$ we have

$$
x_{2}(s)=x_{1}(\theta(s))
$$

for some $C^{2}$-diffeomorphism $\theta:[0,1] \rightarrow[0,1]$ with

$$
\text { either } \theta(0)=0, \theta(1)=1, \quad \text { or } \quad \theta(0)=1, \theta(1)=0 .
$$

Now, $x_{1}$ and $x_{2}$ satisfy the differential equation

$$
\frac{\mathrm{D}}{\mathrm{ds}} \dot{\mathrm{x}}=0 .
$$

Moreover, $\dot{x}_{2}(s)=\dot{\theta}(s) \dot{x}_{1}(\theta(s))$ and

$$
\frac{\mathrm{D}}{\mathrm{d} s} \dot{x}_{2}=\ddot{\theta}(\mathrm{s}) \dot{x}_{1}(\theta(\mathrm{s}))+(\dot{\theta}(\mathrm{s}))^{2} \frac{\mathrm{D}}{\mathrm{d} s} \dot{x}_{1}(\theta(\mathrm{s}))=\ddot{\theta}(\mathrm{s}) \dot{x}_{1}(\theta(\mathrm{s})) .
$$

Therefore, it must be $\ddot{\theta}(s) \dot{x}_{1}(\theta(s))=0$ and since $\dot{x}_{1}(\theta(s)) \neq 0$ for all $s$, we have $\ddot{\theta}(s)=0$ for all s. Then by (6.9) we deduce that either $\theta(s)=s$ or $\theta(s)=1-s$ : in both cases $\mathcal{F}\left(x_{2}\right)=\mathcal{F}\left(x_{1}\right)$, proving $(6.7)$. 


\section{APPENDIX A. ON THE TRANSVERSALITY OF THE NORMAL BUNDLE AND THE TANGENT BUNDLE OF SUBMANIFOLDS}

Let $(M, g)$ be a Riemannian manifold, and let $S \subset M$ be an embedded submanifold. For the application we aim at, $S$ will be the boundary of $M$.

The normal bundle of $S$ will be denoted by $\mathcal{N}(S)$, and for $p \in S$, we set $\mathcal{N}_{p}(S)=\mathcal{N}(S) \cap$ $\mathrm{T}_{\mathrm{p}} M$. The normal bundle $\mathcal{N}(\mathrm{S})$ and the tangent bundle TS will be considered submanifolds of the tangent bundle TM. Given a point $p \in S$ and a normal vector $v \in \mathcal{N}_{p}(S)$, we will denote by $\alpha_{p}^{S}: T_{p} S \times T_{p} S \rightarrow \mathcal{N}_{p}(S)$ the second fundamental form of $S$ at $p$, and by $A_{v}^{S}: T_{p} S \rightarrow T_{p} S$ the shape operator of $S$ at $p$ in the direction $v$, defined by:

$$
g\left(A_{v}^{S}(u), u^{\prime}\right)=-g\left(\alpha^{S}\left(u, u^{\prime}\right), v\right), \quad \forall u, u^{\prime} \in T_{p} S .
$$

For $v \in \mathrm{T}_{p} M$, let us identify $\mathrm{T}_{v}(\mathrm{~T} M)$ with $\mathrm{T}_{\mathrm{p}} M \oplus \mathrm{T}_{\mathrm{p}} M$, with the first summand being the horizontal space of the Levi-Civita connection of $g$ and the second summand being the vertical subspace.

\section{A.1. A transversality condition.}

Proposition A.1. Let $S_{1}, S_{2} \subset M$ hypersurfaces that meet transversally at $p \in S_{1} \cap S_{2}$. Assume that $v \in \mathrm{T}_{\mathrm{p}} \mathrm{M} \backslash\{0\}$ belongs to the intersection $\mathcal{N}\left(\mathrm{S}_{1}\right) \cap \mathrm{TS}_{2}$ and denote by $\mathcal{A}_{v} \subset$ $\mathrm{T}_{\mathrm{p}} \mathrm{M}$ the subspace:

$$
\mathcal{A}_{v}=\left\{A_{v}^{\mathrm{S}_{1}}(w)-\alpha^{\mathrm{S}_{2}}(w, v): w \in \mathrm{T}_{\mathrm{p}} \mathrm{S}_{1} \cap \mathrm{T}_{\mathrm{p}} \mathrm{S}_{2}\right\} .
$$

An element $\left(z, z^{\prime}\right) \in \mathrm{T}_{\mathrm{p}} \mathrm{M} \oplus \mathrm{T}_{\mathrm{p}} \mathrm{M} \cong \mathrm{T}_{v}(\mathrm{TM})$ belongs to the sum $\mathrm{T}_{v}\left(\mathcal{N}_{\mathrm{p}}\left(\mathrm{S}_{1}\right)\right)+\mathrm{T}_{v}\left(\mathrm{TS}_{2}\right)$ iff $z^{\prime}$ belongs to the affine subspace of $\mathrm{T}_{\mathrm{p}} \mathrm{M}$ :

$$
\left[A_{v}^{1}\left(u_{1}\right)+\alpha_{p}^{2}\left(u_{2}, v\right)\right]+\mathcal{A}_{v}+\mathrm{T}_{\mathrm{p}} \mathrm{S}_{2}
$$

where $\mathrm{u}_{1} \in \mathrm{T}_{\mathrm{p}} \mathrm{S}_{1}, \mathrm{u}_{2} \in \mathrm{T}_{\mathrm{p}} \mathrm{S}_{2}$ are chosen in such a way that $z=\mathrm{u}_{1}+\mathrm{u}_{2}$. In particular, $\mathcal{N}\left(\mathrm{S}_{1}\right)$ and $\mathrm{TS}_{2}$ meet transversally at $\mathrm{v}$ if and only if:

$$
\mathcal{A}_{v} \not \subset \mathrm{T}_{\mathrm{p}} \mathrm{S}_{2} \text {. }
$$

Proof. Using the identification $\mathrm{T}_{v}(\mathrm{TM}) \cong \mathrm{T}_{\mathrm{p}} \mathrm{M} \oplus \mathrm{T}_{\mathrm{p}} \mathrm{M}$, the tangent spaces $\mathrm{T}_{v}\left(\mathcal{N}\left(\mathrm{S}_{1}\right)\right)$ and $\mathrm{T}_{v}\left(\mathrm{TS}_{2}\right)$ are given by:

$$
\begin{aligned}
& \mathrm{T}_{v}\left(\mathcal{N}\left(\mathrm{S}_{1}\right)\right)=\left\{\left(\mathrm{u}_{1}, \mathrm{~A}_{v}^{\mathrm{S}_{1}}\left(\mathrm{u}_{1}\right)+\lambda \cdot v\right): \mathrm{u}_{1} \in \mathrm{T}_{\mathrm{p}} \mathrm{S}_{1}, \lambda \in \mathbb{R}\right\}, \\
& \mathrm{T}_{v}\left(\mathrm{TS}_{2}\right)=\left\{\left(\mathrm{u}_{2}, \mathrm{u}_{2}^{\prime}+\alpha^{S_{2}}\left(\mathrm{u}_{2}, v\right)\right): \mathrm{u}_{2}, \mathrm{u}_{2}^{\prime} \in \mathrm{T}_{\mathrm{p}} \mathrm{S}_{2}\right\} .
\end{aligned}
$$

From these equalities, one obtains readily a proof of the first statement.

As to the transversality of $\mathcal{N}\left(S_{1}\right)$ and $\mathrm{TS}_{2}$ at $v$, i.e., $\mathrm{T}_{v}\left(\mathcal{N}\left(\mathrm{S}_{1}\right)\right)+\mathrm{T}_{v}\left(\mathrm{TS}_{2}\right)=\mathrm{T}_{v}(\mathrm{TM})$, the proof follows from a linear algebra argument (apply next Lemma to $V=T_{p} M, V_{1}=$ $\mathrm{T}_{\mathrm{p}} \mathrm{S}_{1}, \mathrm{~V}_{2}=\mathrm{T}_{\mathrm{p}} \mathrm{S}_{2}, \widetilde{\mathrm{V}}_{2}=\mathbb{R} \cdot v, A=A_{v}^{S_{1}}$ and $\left.\alpha=\alpha^{S_{2}}(\cdot, v)\right)$.

Lemma A.2. Let $\mathrm{V}$ be a finite dimensional vector space, and let $\mathrm{V}_{1}, \mathrm{~V}_{2} \subset \mathrm{V}$ be subspaces with $\mathrm{V}_{1}+\mathrm{V}_{2}=\mathrm{V}$, and $\mathrm{V}_{2}$ of codimension 1 . Let $\widetilde{\mathrm{V}}_{2} \subset \mathrm{V}_{2}$ be an arbitrary subspace, let $\mathrm{A}: \mathrm{V}_{1} \rightarrow \mathrm{V}_{1}$ and $\alpha: \mathrm{V}_{2} \rightarrow \mathrm{V}$ be linear maps, and define subspaces $\mathrm{W}_{1}, \mathrm{~W}_{2} \subset \mathrm{V} \oplus \mathrm{V}$ and $\mathcal{A} \subset \vee$ by:

$$
\begin{aligned}
& W_{1}=\left\{\left(u_{1}, A\left(u_{1}\right)+\widetilde{v}_{2}\right): u_{1} \in v_{1}, \widetilde{v}_{2} \in \widetilde{v}_{2}\right\}, \\
& W_{2}=\left\{\left(u_{2}, v_{2}+\alpha\left(u_{2}\right)\right): u_{2}, v_{2} \in v_{2}\right\}, \\
& \mathcal{A}=\left\{A w-\alpha w: w \in v_{1} \cap v_{2}\right\} .
\end{aligned}
$$

Then, $\mathrm{W}_{1}+\mathrm{W}_{2}=\mathrm{V} \oplus \mathrm{V}$ if and only if $\mathcal{A} \not \subset \mathrm{V}_{2}$. 
Proof. The equality $W_{1}+W_{2}=V \oplus V$ holds iff for all $s, t \in V$, there exist $u_{1} \in V_{1}$, $\widetilde{v}_{2} \in \widetilde{\mathrm{V}}_{2}$ and $\mathrm{u}_{2}, v_{2} \in \mathrm{V}_{2}$ such that:

$$
u_{1}+u_{2}=s, \quad \text { and } A\left(u_{1}\right)+\widetilde{v}_{2}+v_{2}+\alpha\left(u_{2}\right)=t .
$$

Since $V_{1}+V_{2}=V$, there exist $\bar{u}_{1} \in V_{1}$ and $\bar{u}_{2} \in V_{2}$ such that $\bar{u}_{1}+\bar{u}_{2}=s$; every other pair $\left(u_{1}, u_{2}\right) \in V_{1} \times V_{2}$ with $u_{1}+u_{2}=s$ is of the form $u_{1}=\bar{u}_{1}+h$ and $u_{2}=\bar{u}_{2}-h$ for some $h \in V_{1} \cap V_{2}$. Thus, the desired transversality holds iff there exists $h \in V_{1} \cap V_{2}$, $\widetilde{v}_{2} \in \widetilde{V}_{2}$ and $v_{2} \in V_{2}$ such that:

$$
A\left(\bar{u}_{1}\right)+A(h)+\widetilde{v}_{2}+v_{2}+\alpha\left(\bar{u}_{2}\right)-\alpha(h)=t,
$$

i.e.,

$$
A(h)-\alpha(h)+v_{2}=t-\widetilde{v}_{2}-A\left(\bar{u}_{1}\right)-\alpha\left(\bar{u}_{2}\right) .
$$

Clearly, when $t$ is arbitrary in $V$, the right hand side of the above equality is an arbitrary vector in $V$. Thus, $W_{1}+W_{2}=V \oplus V$ holds iff the linear map

$$
\left(\mathrm{V}_{1} \cap \mathrm{V}_{2}\right) \times \mathrm{V}_{2} \ni\left(\mathrm{h}, v_{2}\right) \longmapsto \mathrm{A}(\mathrm{h})-\alpha(\mathrm{h})+v_{2} \in \mathrm{V}
$$

is surjective. Since $V_{2}$ has codimension 1 in $V$, this holds iff the image of the linear map $V_{1} \cap V_{2} \ni h \mapsto A(h)-\alpha(h) \in V$ is not contained in $V_{2}$, i.e., iff $\mathcal{A} \not \subset V_{2}$.

A.2. The case of a 1-parameter family of submanifolds. We will now study a transversality problem between the tangent bundle of a hypersurface $S_{2}$, and the union of the normal bundles of a smooth 1 -parameter family of hypersurfaces $\left.S_{1}(t), t \in\right]-\varepsilon, \varepsilon[$.

More precisely, let us consider the following setup.

- $S_{1}, S_{2} \subset M$ are hypersurfaces, and $p \in S_{1} \cap S_{2}$;

- there exists a nonzero $v \in \mathcal{N}_{p}\left(S_{1}\right) \cap T_{p} S_{2}$. In particular, $S_{1}$ and $S_{2}$ meet transversally at $p$.

- $\mathbf{n}$ is a smooth unit vector field along $S_{1}$;

- $F$ : $]-\varepsilon, \varepsilon\left[\times S_{1} \rightarrow M\right.$ is defined by:

$$
F(t, x)=\exp _{x}(t \cdot \mathbf{n}(x))
$$

- $S_{1}(t)$ is defined as the image of the map $F(t, \cdot): S_{1} \rightarrow M$.

Under suitable non-focality assumption for $p$, we can assume that $F$ is an embedding of ]$-\varepsilon, \varepsilon\left[\times S_{1}\right.$ onto an open subset of $M$. Its image is foliated by the smooth hypersurfaces $S_{1}(t)$.

We consider the union:

$$
\bigcup_{t \in]-\varepsilon, \varepsilon[} \mathcal{N}\left(S_{1}(t)\right) \subset T M
$$

this is a smooth submanifold of TM. Under the above assumptions, $\frac{\partial F}{\partial t}(t, x)$ is a unit vector normal to $S_{1}(t)$ at $F(t, x)$, and we have a parameterization of the union $\bigcup_{t \in]-\varepsilon, \varepsilon[} \mathcal{N}\left(S_{1}(t)\right)$ given by the smooth map:

$$
\mathrm{G}:]-\varepsilon, \varepsilon\left[\times S_{1} \times \mathbb{R} \longrightarrow \mathrm{TM}\right.
$$

defined by:

$$
\left.G(t, y, \lambda)=\lambda \cdot \frac{\partial F}{\partial t}(t, y), \quad(t, y, \lambda) \in\right]-\varepsilon, \varepsilon\left[\times S_{1} \times \mathbb{R} .\right.
$$

Our aim is to determine when the map $G$ is transversal in $\operatorname{TM}$ to $\mathrm{TS}_{2}$ at the point $(0, p, \lambda)$, where $\mathrm{G}(0, \mathrm{p}, \lambda)=\lambda \cdot \mathbf{n}(\mathrm{p})=\nu$. 
Clearly, the image of $\mathrm{dG}(0, p, \lambda)$ is given by:

$$
\operatorname{Im}(\mathrm{dG}(0, p, \lambda))=\mathbb{R} \cdot \frac{\partial \mathrm{G}}{\partial \mathrm{t}}(0, p, \lambda)+\mathrm{T}_{v}\left(\mathcal{N}\left(\mathrm{S}_{1}\right)\right) .
$$

As we have observed above, if $\mathrm{T}_{v}\left(\mathcal{N}\left(\mathrm{S}_{1}\right)\right)+\mathrm{T}_{v}\left(\mathrm{TS}_{2}\right)=\mathrm{T}_{v}(\mathrm{TM})$, i.e., if $\mathcal{A}_{v} \not \subset \mathrm{T}_{\mathrm{p}} \mathrm{S}_{2}$, then $\mathrm{G}$ is transversal to $\mathrm{TS}_{2}$ at $(0, p, \lambda)$.

On the other hand, if $\mathrm{T}_{v}\left(\mathcal{N}\left(\mathrm{S}_{1}\right)\right)+\mathrm{T}_{v}\left(\mathrm{TS}_{2}\right) \neq \mathrm{T}_{v}(\mathrm{TM})$, then it is easy to check that $\mathrm{T}_{v}\left(\mathcal{N}\left(\mathrm{S}_{1}\right)\right)+\mathrm{T}_{v}\left(\mathrm{TS}_{2}\right)$ has codimension 1 in $\mathrm{T}_{v}(\mathrm{TM})$. This follows immediately from the observation the the projection onto the first factor $T_{v}(T M) \cong T_{p} M \times T_{p} M \rightarrow T_{p} M$ induces an isomorphism between the quotient:

$$
\left(\mathrm{T}_{v}\left(\mathcal{N}\left(\mathrm{S}_{1}\right)\right)+\mathrm{T}_{v}\left(\mathrm{TS}_{1}\right)\right) /\left(\{0\} \times \mathrm{T}_{\mathrm{p}} \mathrm{S}_{2}\right)
$$

and $T_{p} M$.

Thus, $\mathrm{G}$ is transversal to $\mathrm{TS}_{2}$ at $(0, p, \lambda)$ iff

$$
\frac{\partial \mathrm{G}}{\partial \mathrm{t}}(0, \mathrm{p}, \lambda) \notin \mathrm{T}_{v}\left(\mathcal{N}\left(\mathrm{S}_{2}\right)\right)+\mathrm{T}_{v}\left(\mathrm{TS}_{1}\right)
$$

Now, $\frac{\partial G}{\partial t}(0, p, \lambda)=(\mathbf{n}(p), 0)$, because the $t \mapsto \frac{\partial F}{\partial t}(t, p)$ is parallel along the geodesic $\mathrm{t} \mapsto \mathrm{F}(\mathrm{t}, \mathrm{p})$. Since $v=\lambda \cdot \mathbf{n}(\mathrm{p})$, we conclude that $\mathrm{G}$ is transversal to $\mathrm{TS}_{2}$ at $(0, p, \lambda)$ iff $(v, 0) \notin \mathrm{T}_{v}\left(\mathcal{N}\left(\mathrm{S}_{1}\right)\right)+\mathrm{T}_{v}\left(\mathrm{TS}_{1}\right)$. This happens iff $\alpha_{\mathrm{p}}^{2}(v, v) \notin \mathrm{T}_{\mathrm{p}} \mathrm{S}_{2}$, i.e., iff $\alpha_{\mathrm{p}}^{2}(v, v) \neq 0$.

In conclusion, we have proved the following:

Proposition A.3. In the above notations, the submanifold

$$
\bigcup_{t \in]-\varepsilon, \varepsilon[} \mathcal{N}\left(S_{1}(t)\right)
$$

is transversal to $\mathrm{TS}_{2}$ at $v$ iff either one of the two conditions holds:

(a) $\mathcal{A}_{v} \not \subset \mathrm{T}_{\mathrm{p}} \mathrm{S}_{2}$;

(b) $\alpha_{p}^{2}(v, v) \neq 0$

Note that conditions (a) and (b) above are stable by $\mathrm{C}^{2}$-small perturbations of the metric $\mathrm{g}$.

Corollary A.4. Let $\mathrm{N} \geqslant 2$ and let $\mathbb{D}^{\mathrm{N}}$ be the $\mathrm{N}$-dimensional unit disk in $\mathbb{R}^{n}$. The set of Riemannian metrics on $\mathbb{D}^{\mathrm{N}}$ that admit $O-T$ chords has nonempty interior relatively to the $\mathrm{C}^{2}$-topology.

Proof. Given a metric $g$ on $\mathbb{D}^{\mathrm{N}}$, an $\mathrm{O}-\mathrm{T}$ chord in $\mathbb{D}^{\mathrm{N}}$ relative to $\mathrm{g}$ corresponds to the intersection of the tangent bundle of some open portion $\mathrm{S}_{2}$ of $\partial \mathrm{D}^{\mathrm{N}}$ and the normal bundle of some other open portion $S_{1}$ of $\partial D^{N}$. Evidently, one can easily construct a (smooth) metric $\mathrm{g}$ for which such intersection exists, and for which either one of conditions (a) and (b) of Proposition A.3 holds. For instance, condition (b) is rather easy to obtain. In this situation, the transversality implies that sufficiently $C^{2}$-small perturbations of the metric will preserve the existence of some intersection between the normal and the tangent bundle of $\partial D^{N}$. This says that $\mathrm{O}-\mathrm{T}$ chords will persist by sufficiently $\mathrm{C}^{2}$-small perturbations of the metric, i.e., the set of metrics in $\mathbb{D}^{\mathrm{N}}$ admitting $\mathrm{O}-\mathrm{T}$ chords has nonempty interior.

It is not hard to see that, in fact, the conclusion of Corollary A.4 holds more generally for arbitrary smooth (compact) manifolds with boundary, whose dimension is greater than or equal to 2 . 


\section{REFERENCES}

[1] W. Bos, Kritische Sehenen auf Riemannischen Elementarraumstücken, Math. Ann. 151 (1963), 431-451.

[2] A. CANino On p-convex sets and geodesics, J.Diff.Eq. 75, (1988), 118-157.

[3] A. Canino, M. Degiovanni Nonsmooth critical point theory and quasilinear elliptic equations, Proc. Topological Methods in Differential Equations and Inclusions, A.Granas and M.Frigon ed., Montreal 1994, $1-50$.

[4] J:-N. Corvellec, M. Degiovanni, M: Marzocchi Deformation properties for continuos functionals adn critical point theory, Top. Meth. Nonlinerar Anal., 1, (1993), 151-171

[5] E. De Giorgi, A. Marino. M. Tosques Problemi di evoluzione in spazi metrici e curve di massima pendenza, Atti Accad. Naz. Lincei Rend. Cl. Sci. Fis. Mat. Natur. (8) 68 (1980), 180-187.

[6] M. Degiovanni, A. Marino. M. Tosques Evolution equation with lack of convexity Nonlin. Anal. 9 (1985), 1401-1443.

[7] M. Degiovanni, M. Marzocchi, A Critical Point Theory for Nonsmooth Functionals, Ann. Mat. Pura Appl. 167 (1994), 73-100.

[8] E. FAdell, AND S. Hus SEINI, Relative cohomological index theories, Adv. Math 64 (1987), 1-31.

[9] G. Fournier, AND M. Willem, Multiple solutions of the forced double pendulum equation, Ann. Inst. H. Poincaré, Analyse non Lineaire 6 (suppl.) (1989), 259-281.

[10] R. Giambò, F. Giannoni, P. Piccione, Orthogonal Geodesic Chords, Brake Orbits and Homoclinic Orbits in Riemannian Manifolds, Adv. Diff. Eq., 10, (2005), pp 931-960.

[11] _ Existence of orthogonal geodesic chords on Riemannian manifolds with concave boundary and homeomorphic to the $\mathrm{N}$-dimensional disk, Nonlinear Analysis Series A: Theory, Methods, Applications, 73 (2010) 290-337.

[12] _ Multiple Brake Orbits and Homoclinics in Riemannian Manifolds, Archive for Rational Mechanics and Analysis: 200, (2011), 691-724.

[13] _ Examples with minimal number of brake orbits and homoclinics in annular potential regions, J. Differential Equations 256 (2014), 2677-2690

[14] _ Multiple brake orbits in m-dimensional disks, Calc. Var. 54 (2015), 2553-2580.

[15] _ Functions on the sphere with critical points in pairs and orthogonal geodesic chords, J. Differential Equations, 260, (2016), 8261-8275.

[16] D. Gilbarg, N. S. TRUdinger, Elliptic partial differential equations of second order, Springer-Verlag, Berlin, (1983)

[17] H. LiU, Y. LONG, Resonance identity for symmetric closed characteristics on symmetric convex Hamiltonian energy hypersurfaces and its applications J. Differential Equations 255 (2013), 2952-2980.

[18] C. LiU, D. Zhang, Seifert conjecture in the even convex case, Comm. Pure and Applied Math. 67 (2014), 1563-1604.

[19] Y. Long, D. ZhANG, C. ZhU, Multiple brake orbits in bounded convex symmetric domains, Adv. Math. 203 (2006), no. 2, 568-635.

[20] Y. Long, C. ZHU, Closed characteristics on compact convex hypersurfaces in $\mathbb{R}^{2 n}$ Ann. of Math. (2) 155 (2002), no. 2, 317-368.

[21] L. Lusternik, L. Schnirelman, Methodes Topologiques dans les Problemes Variationelles, Hermann, 1934.

[22] A. Marino, D. Scolozzi, Geodetiche con ostacolo Boll. U.M.I., (6) 2-B (1983), 1-31.

[23] P. H. Rabinowitz, Critical point theory and applications to differential equations: a survey. Topological nonlinear analysis, Progr. Nonlinear Differential Equations Appl., 15, Birkhauser Boston, Boston, MA, (1995), 464-513.

[24] D. ScolozzI, Esistenza e molteplicitá di geodetiche con ostacolo e con estremi variabili, Ricerche Mat. 33 (1984), 171-201.

[25] D. ScolozzI, Un risultato di locale unicitá per le geodetiche su varietá con bordo, Boll. U.M.I., (6) 5-B (1986), 309-327.

[26] H. SeIFERT, Periodische Bewegungen Machanischer Systeme, Math. Z. 51 (1948), 197-216.

[27] H. Whitney, The self-intersections of a smooth $n$-manifold in 2n-space, Ann. of Math. (2) 45, (1944), 220-246.

[28] D. ZHANG, Brake type closed characteristics on reversible compact convex hypersurfaces in $\mathbb{R}^{2 n}$ Nonlinear Analysis 74 (2011) 3149-3158.

[29] D. ZhANG, C.LiU, Multiplicity of brake orbits on compact convex symmetric reversible hypersurfaces in $\mathbb{R}^{2 n}$ for $\mathrm{n} \geqslant 4$, Proc. London Math. Soc. (3) 107 (2013) 1-38 
[30] - Multiple brake orbits on compact convex symmetric reversible hypersurfaces in $\mathbb{R}^{2 n}$, Ann. Inst. H. Poincar 'e Anal. Non Linéaire 31 (2014), no. 3, 531-554.

UNIVERSITÀ DI CAMERINO

Scuola di Scienze E TeCNOLOGIE

CAMERINO (MC)

ITALY

E-mail address: roberto.giambo@unicam.it fabio.giannoni@unicam.it
Universidade de São PaUlo

Departamento de Matemática

SÃo PAULO, SP

BRAZIL

E-mail address: piccione@ime.usp.br 